\title{
Role of boundary conditions on Lifshitz spacetimes
}

\author{
Lissa de Souza Campos $\odot,{ }^{1,2, *}$ Claudio Dappiaggi $\oplus^{1,2, \dagger}$ and Denis Sina $\oplus^{1, \$}$ \\ ${ }^{1}$ Dipartimento di Fisica, Università degli Studi di Pavia, Via Bassi, 6, 27100 Pavia, Italy \\ ${ }^{2}$ Istituto Nazionale di Fisica Nucleare-Sezione di Pavia, Via Bassi, 6, 27100 Pavia, Italy
}

(Received 23 April 2021; accepted 12 October 2021; published 10 November 2021)

\begin{abstract}
On a class of four-dimensional Lifshitz spacetimes with critical exponent $z=2$, including a hyperbolic and a spherical Lifshitz topological black hole, we consider a real Klein-Gordon field. Using a mode decomposition, we split the equation of motion into a radial and into an angular component. As first step, we discuss under which conditions on the underlying parameters we can impose to the radial equation boundary conditions of Robin type and whether bound state solutions do occur. Subsequently, we show that, whenever bound states are absent, one can associate to each admissible boundary condition a ground and a KMS state whose associated two-point correlation function is of local Hadamard form.
\end{abstract}

DOI: 10.1103/PhysRevD.104.105008

\section{INTRODUCTION}

In the quest of finding a quantization scheme for the gravitational field, Hořava-Lifshitz gravity, [1], was first introduced as a theory in which the underlying spacetime possesses a time coordinate $t$ and spatial counterparts $\underline{x}$ appearing with a different scaling behavior

$t \mapsto \delta^{z} t$ and $\underline{x} \mapsto \delta \underline{x}$ with $z>1$ and $\delta>0$.

While we will not focus on the developments towards a quantum theory of gravitation, referring an interested reader to the recent review [2], we stress that also different applications of these models have been discussed ranging from cosmology, to quantum critical systems [3], to condensed matter physics, see e.g., [4] and references therein, and to the AdS/CFT correspondence.

Especially motivated from this last framework, a particular interest has been devoted to studying the behavior both at a classical and at a quantum level of a scalar field living either on a Lifshitz spacetime or on asymptotically Lifshitz black holes [5-7]. A remarkable property of all these manifolds lies in the fact that the underlying metric possesses a singular behavior along a spatial direction and therefore the spacetime is not globally hyperbolic. As a consequence, the dynamics of a scalar field cannot be fully

\footnotetext{
*lissa.desouzacampos01@universitadipavia.it

†claudio.dappiaggi@unipv.it

¥denis.sina01@universitadipavia.it
}

Published by the American Physical Society under the terms of the Creative Commons Attribution 4.0 International license. Further distribution of this work must maintain attribution to the author(s) and the published article's title, journal citation, and DOI. Funded by SCOAP. determined only by assigning initial data, but one needs also to specify an asymptotic behavior towards the singularity of the metric, exactly as it occurs when one considers Lorentzian manifolds with a timelike boundary, such as asymptotically anti-de Sitter spacetimes. A close scrutiny of the literature unveils that, in the case of backgrounds of Lifshitz type, only Dirichlet boundary conditions have been considered.

Yet, in the past few years, starting from the work of Ishibashi and Wald $[8,9]$ on the dynamics of free Bosonic fields on anti-de Sitter spacetimes, it has become clear that one can consider a much larger class of boundary conditions of Robin type, yielding well-defined dynamics and, in addition, admitting an associated, full-fledged quantization scheme. This enlarged set of options has been studied in detail on anti-de Sitter spacetime [10-12], on a rotating BTZ black hole $[13,14]$ and recently on massless hyperbolic black holes [15]. From a technical viewpoint the implementation of this framework amounts to considering only self-adjoint extensions of the radial part of the KleinGordon operator and to work with the square-integrable eigenfunctions of such operator. From a physical viewpoint, these boundary conditions are noteworthy also because they imply that we are ultimately considering a closed system, namely the flux of energy and momentum across the Lifshitz boundary vanishes, see in particular [9].

Inspired by these works, we consider a massive, real, scalar field, with an arbitrary coupling to scalar curvature and we investigate whether one can extend the class of admissible boundary conditions on a four dimensional Lifshitz spacetime with dynamical critical exponent $z=2$ and on its generalizations to a hyperbolic and to a spherical Lifshitz topological black hole, as determined in [16]. Our analyses expand the results of Sec. III of [6], where a correlation function on pure Lifshitz spacetimes 
was constructed, by considering massive fields instead of massless, general Robin boundary conditions instead of Dirichlet, and two Lifshitz spacetimes beyond an exact Lifshitz geometry. It is worth mentioning that, within holographic theories, one is often interested in considering energy and momentum sources at the boundary or other Lifshitz bulk spacetimes. In these cases, one may consider either generalizing the analysis we performed or working within other frameworks such as done in [17].

Without entering here into the technical details of the analysis, our investigation leads to two main results. On the one hand, we prove that, in all these spacetimes, there exists a specific range of the underlying parameters, namely the mass and the coupling to scalar curvature, for which a general class of boundary conditions of Robin type is admissible. In this respect, it is worth emphasizing that our result is compatible with [18]. On the other hand, we show that, similarly to what happens on anti-de Sitter spacetimes, one can stumble into the so-called bound state modes. More precisely, since all the spacetimes that we consider are static, it is possible to study the dynamics of the underlying field and the implementation of the boundary conditions by considering the Fourier transform along the time coordinate $t$. As a consequence one can show that, depending on the range of boundary conditions considered, $\omega$, the Fourier parameter associated to $t$, is not necessarily real but it can also take value in a discrete set of purely imaginary frequencies, the above mentioned bound states modes. Here we confirm and extend the results of [19], in which it was shown the occurrence of instabilities for a scalar field on Lifshitz spacetime for a specific range of the field parameters. Although at a classical level, this feature is not particularly problematic, it has more severe consequences at a quantum level since it entails the existence of modes, exponentially growing in time. As a consequence, whenever such bound states do appear, it is not possible to construct the two-point function of a ground state associated to the underlying scalar field, as already observed in [10].

After having established in which range of boundary conditions bound states do not occur, in the remaining part of the paper we focus our attention on all other cases and we show how to construct for each of them a two-point correlation function both for a ground state and for a KMS/ thermal state at arbitrary temperature. It is important to observe that all these two-point functions obey the underlying equation of motion, they implement the canonical commutation relations as well as the chosen boundary conditions and, in addition, they are of local Hadamard form. Such property guarantees the possibility of constructing Wick-ordered observables which is an important prerequisite for studying interactions at the level of perturbation theory as well as the back-reaction induced by a regularized stress-energy tensor.

The content of the paper is as follows. First, in Sec. II, we review the geometric data of the class of spacetimes of
Lifshitz type that we considered. Secondly, in Sec. III, we consider the Klein-Gordon equation and we show that it can be reduced to a radial equation by a mode decomposition of its solutions. Then, we study the radial equation together with its associated Green function, separately on Lifshitz spacetime, on a hyperbolic and on a spherical Lifshitz black hole, respectively, in Secs. IV, V, and VI. In Sec. VII, we construct on each spacetime considered and for each admissible boundary condition the two-point function both of the ground and of a KMS/thermal state. Several computations of this work are reproduced in a Mathematica notebook available at [20].

\section{HOŘAVA-LIFSHITZ SOLUTIONS}

In the following we call $\mathcal{R}$ the Ricci scalar determined by a metric tensor $g$ on a four dimensional Lorentzian spacetime $\mathcal{M}$ while $L$ is scale length related to the cosmological constant via $\Lambda=-\left.\frac{z^{2}+z+4}{2 L^{2}}\right|_{z=2}=-\frac{5}{L^{2}}$. In addition we indicate with $\varepsilon^{\mu \nu \alpha \beta}$ the Levi-Civita tensor density, with $F_{\mu \nu}=$ $\left.\partial_{[\mu} A_{\nu}\right]$ and $\left.H_{\mu \nu \tau}=\partial_{[\mu} B_{\nu}\right]$ two Abelian gauge fields, while $C=\frac{2}{L}$ is a constant, coupling parameter. As shown in [16], four-dimensional Lifshitz topological black holes $\left(\mathrm{Lif}_{\kappa}, g_{\kappa}\right)$, where $\kappa \in\{-1,0,1\}$ are solutions of the Euler-Lagrange equations associated to the action

$$
\begin{aligned}
S= & \int_{\mathcal{M}} d^{4} x \sqrt{|g|}\left(\mathcal{R}-2 \Lambda-\frac{1}{4} F_{\mu \nu} F^{\mu \nu}-\frac{1}{12} H_{\mu \nu \tau} H^{\mu \nu \tau}\right. \\
& \left.-\frac{C}{\sqrt{|g|}} \varepsilon^{\mu \nu \alpha \beta} B_{\mu \nu} F_{\alpha \beta}\right)
\end{aligned}
$$

where, given a global coordinate chart $(t, r, \theta, \varphi)$,

$$
F_{r t}=2 L r \quad \text { and } \quad H_{r \theta \varphi}=2 L^{2} r j_{\kappa}(\theta),
$$

with

$$
j_{\kappa}(\theta):= \begin{cases}\theta, & \kappa=0 \\ \sinh (\theta), & \kappa=-1, \\ \sin (\theta), & \kappa=+1\end{cases}
$$

All other components of $F$ or $H$ are either determined by these via antisymmetrization or they are vanishing. Here $t$ runs over the whole real line, $(\theta, \varphi)$ are the standard coordinates over the 2-plane, the 2-hyperboloid, and the 2-sphere, respectively, for $\kappa=0, \kappa=-1$ and $\kappa=1$. Also, $r \in(0, \infty)$ if $\kappa=0$ or $\kappa=1$, and $r \in(L / \sqrt{2}, \infty)$ if $\kappa=-1$. For $\kappa \in\{-1,0,+1\}$, their line element reads:

$$
\begin{aligned}
d s^{2}= & -\frac{r^{2}}{L^{2}}\left(\frac{r^{2}}{L^{2}}+\frac{\kappa}{2}\right) d t^{2}+\left(\frac{r^{2}}{L^{2}}+\frac{\kappa}{2}\right)^{-1} d r^{2}+r^{2} d \theta^{2} \\
& +r^{2} j_{\kappa}(\theta)^{2} d \varphi^{2} .
\end{aligned}
$$


All of them are static, nonglobally hyperbolic and geodesically incomplete. For $\kappa=0, \kappa=-1$ and $\kappa=+1$, the line element (2.3) corresponds to, respectively, a flat, a hyperbolic, and a spherical Lifshitz topological black hole. The Lifshitz horizon $r=0$ is a coordinate singularity for $\kappa=0$, but for $\kappa \neq 0$, the Ricci and the Kretschmann scalars diverge there. In the hyperbolic case, there is a horizon at $r=L / \sqrt{2}$, while in the spherical case, $r=0$ is a naked singularity. Comparing them with topological black holes within Einstein gravity [21], we observe that compact sections at fixed $r$ and $t$ can also be obtained for Lifshitz topological black holes by suitable identifications in the spatial coordinates. In contrast, a naked singularity for the spherical black hole is present only in the Lifshitz case.

Furthermore, note that a flat topological black hole $\left(\mathrm{Lif}_{0}, g_{0}\right)$ is equivalent to a Lifshitz spacetime with critical exponent $z=2$ and with polar coordinates for the sections of constant time and radius. Hence, it also solves EinsteinMaxwell-Dilaton and Einstein-Proca gravity theories, as detailed in ([22], pg. 27). In addition, $\left(\right.$ Lif $\left._{0}, g_{0}\right)$ satisfies the scaling relations (1.1) characteristic of Hořava-Lifshitz gravity with $z=2$, which in the coordinates of Eq. (2.4) read

$$
\begin{aligned}
t & \rightarrow \delta^{2} t, \quad r \rightarrow \delta^{-1} r, \\
\theta & \rightarrow \delta \theta \quad \text { and } \quad \varphi_{1}, \ldots \varphi_{n-3} \rightarrow \varphi_{1}, \ldots \varphi_{n-3} .
\end{aligned}
$$

As $r \rightarrow \infty$, the $g_{t t}$ component of the line element (2.3) diverges faster than the others. This implies that near radial infinity the light cones flatten out and the effective speed of light diverges. Consequently, two distinct points at radial infinity with equal time share the same past and the same future, and, accordingly, we say that these spacetimes are not causally distinguishable at the boundary. An illustration of the causal structure-not a Penrose diagram in the standard sense — of Lifshitz topological black holes is given in Fig. 1. A detailed study regarding the anisotropic conformal infinity, generalizing Penrose's notion of conformal infinity, can be found in [23]. It is worth mentioning that the interpretation of the asymptotic behavior of a Lifshitz spacetime as $r \rightarrow \infty$ is not unique. In fact, other definitions of a Lifshitz boundary can be considered, see e.g., [24]. In this work, however, we stick to the interpretation of the boundary as outlined in Sec. III of [6], i.e., merely as the asymptotic radial infinity for the bulk theory. We reckon that, in this way, it is easier for a reader to compare our analysis to the ones in asymptotically AdS spacetime, e.g., [8-12], which have inspired our approach to the analysis of boundary conditions. For further comments on the metric solutions (2.3), we refer to [16].

\section{KLEIN-GORDON FIELD}

On $\left(\right.$ Lif $\left._{\kappa}, g_{\kappa}\right)$, let us consider a real, massive, scalar field $\Psi: \mathrm{Lif}_{K} \rightarrow \mathbb{R}$ whose action is given by

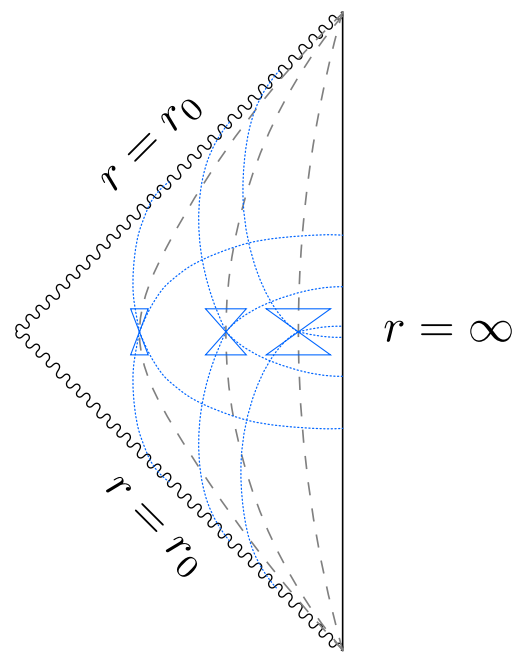

FIG. 1. Diagram representing flat $\left(r_{0}=0\right)$, hyperbolic $\left(r_{0}=L / \sqrt{2}\right)$, and spherical $\left(r_{0}=0\right)$ Lifshitz topological black holes. In all cases we have a singularity at $r=r_{0}$ and a degenerate Lifshitz boundary at $r=\infty$. The dashed lines are static trajectories, and the dotted lines are light rays.

$S=-\frac{1}{2} \int_{\text {Lif }_{\kappa}} d^{4} x \sqrt{\left|g_{\kappa}\right|}\left(\nabla_{\alpha} \Psi \nabla^{\alpha} \Psi+\left(\mu_{0}^{2}+\xi \mathcal{R}\right) \Psi^{2}\right)$,

where $\mu_{0}^{2} \in \mathbb{R}^{+}$is the mass parameter of the scalar field, $\xi \in \mathbb{R}$ is the scalar-curvature coupling constant and the Ricci scalar built out $g_{\kappa}$ is given by:

$$
\mathcal{R}=-\frac{22}{L^{2}}-\frac{\kappa}{r^{2}}
$$

where, with a slight abuse of notation, we omit to indicate explicitly the $\kappa$-dependence of $\mathcal{R}$. For convenience, we define the effective mass $\mu^{2}=\mu_{0}^{2}-\xi \frac{22}{L^{2}} \in \mathbb{R}$. Observe that, due to the anisotropy of the line element, if $\kappa \neq 0$ then we cannot rescale the coordinates to obtain a constant Ricci scalar.

From the action (3.1), it descends the Klein-Gordon equation, which governs the dynamics of the scalar field:

$$
P \Psi=\left(\square-\mu^{2}+\xi \frac{\kappa}{r^{2}}\right) \Psi=0,
$$

where $\square$ is the D'Alembert wave operator built out of the metric $g_{\kappa}$ on $\mathrm{Lif}_{\kappa}$.

In order to solve Eq. (3.3), with line element given by Eq. (2.3), we observe that the metric admits a global timelike Killing field $\partial_{t}$ and the scalar field $\Psi$ can be written in terms of a Fourier expansion with respect to $t$. Moreover, by separation of variables and the superposition principle, we make the following ansatz: 


$$
\begin{aligned}
& \Psi(t, r, \theta, \varphi) \\
& \quad=\int_{\sigma(\Delta)} d \Sigma(\ell, m) \int_{\mathbb{R}} d \omega e^{-i \omega t} R(r) Y_{\ell}^{m}(\theta, \varphi) .
\end{aligned}
$$

The harmonics $Y_{\ell}^{m}(\theta, \varphi)$ are the eigenfunctions of the Laplacian operator $\Delta$ with eigenvalue $\lambda_{\ell}^{m}$. The Laplacian operator comes from the angular part of $\square$ and it depends on the case considered:

$\Delta= \begin{cases}\frac{1}{\theta^{2}} \frac{\partial^{2}}{\partial \varphi^{2}}+\frac{1}{\theta} \frac{\partial}{\partial \theta}+\frac{\partial^{2}}{\partial \theta^{2}}, & \text { for } \kappa=0 ; \\ \frac{1}{\sinh (\theta)^{2}} \frac{\partial^{2}}{\partial \varphi^{2}}+\frac{1}{\sinh (\theta)} \frac{\partial}{\partial \theta}\left(\sinh (\theta) \frac{\partial}{\partial \theta}\right), & \text { for } \kappa=-1 ; \\ \frac{1}{\sin (\theta)^{2}} \frac{\partial^{2}}{\partial \varphi^{2}}+\frac{1}{\sin (\theta)} \frac{\partial}{\partial \theta}\left(\sin (\theta) \frac{\partial}{\partial \theta}\right), & \text { for } \kappa=+1 .\end{cases}$

The integral with measure $d \Sigma(\ell, m)$ over the space $\sigma(\Delta)$ denotes that we are summing over all harmonics, which depend on the quantum numbers $\ell$ and $m$. For the explicit definition of the harmonics, of $d \Sigma(\ell, m)$ and of $\sigma(\Delta)$, we refer the reader to [25] for the flat and spherical cases, and to [26] or to ([15], Appendix A) for the hyperbolic case. On the contrary, since they appear explicitly in the form of the radial component $R(r)$, we report explicitly the form of the eigenvalues $\lambda_{\ell}^{m}$ :

$$
\lambda_{\ell}^{m}= \begin{cases}-\left(\ell^{2}+m^{2}\right), \ell, m \in \mathbb{R}, & \text { for } \kappa=0, \\ -\left(\frac{1}{4}+\ell^{2}\right), \ell \in \mathbb{R}, & \text { for } \kappa=-1, \\ -\ell(\ell+1), \ell \in \mathbb{N}_{0}, & \text { for } \kappa=+1 .\end{cases}
$$

It follows that $\lambda_{\ell}^{m}$ ranges over a discrete spectrum for $\kappa=+1$, while, otherwise, it assumes continuous values on the negative portion of the real line.

Substituting the ansatz (3.4) in Eq. (3.3), the KleinGordon equation reduces to an ordinary differential equation, which we call the radial equation

$$
R^{\prime \prime}(r)+Q(r) R^{\prime}(r)+V(r) R(r)=0,
$$

with

$$
\begin{aligned}
Q(r)= & \frac{4 r}{\kappa L^{2}+2 r^{2}}+\frac{3}{r}, \\
V(r)= & \frac{4 L^{6}}{\left(\kappa L^{2} r+2 r^{3}\right)^{2}} \omega^{2}+\frac{2 L^{2}}{\kappa L^{2} r^{2}+2 r^{4}} \lambda \\
& -\frac{2 L^{2}}{\kappa L^{2}+2 r^{2}} \mu^{2},
\end{aligned}
$$

where

$$
\lambda:=\lambda_{\ell}^{m}+\kappa \xi .
$$

It is noteworthy that the radial potential associated to the Ricci scalar leads solely to a shift of the eigenvalue $\lambda_{\ell}^{m}$ by a factor $\kappa \xi$. For $\kappa=0$ and $\kappa=+1$, the domain of the radial equation is $r \in(0, \infty)$, while for $k=-1$, we have $r \in(L / \sqrt{2}, \infty)$. According to the standard endpoint classification of ordinary differential equations, in all cases, $r=\infty$ is an irregular singular point. The other endpoint is a regular singularity for $\kappa= \pm 1$, while it is an irregular one for $\kappa=0$.

The radial equation can be written in well-known forms: as a hypergeometric equation for $\kappa \neq 0$ and as a confluent hypergeometric equation for $\kappa=0$. To obtain these forms we apply different coordinate changes in each case, hence we treat them separately in the following sections. Before that, some general considerations are in order.

First of all, observe that, since the geometric structure of radial infinity is the same on all $\mathrm{Lif}_{\kappa}$ spacetimes, the solutions of Eq. (3.7) behave similarly asymptotically, namely

$$
R(r) \sim r^{-2 \pm \nu}, \quad \text { as } r \rightarrow \infty,
$$

where

$$
\nu:=\sqrt{4+L^{2} \mu^{2}} .
$$

We require $\nu>0$, which is equivalent to imposing the Breitenlohner-Freedman bound on the effective mass. In addition, observe that negative values of the effective mass encompass

$\mu^{2} \in\left(-\frac{4}{L^{2}}, 0\right) \Leftrightarrow \xi \in\left(\frac{L^{2} \mu_{0}^{2}}{22}, \frac{L^{2} \mu_{0}^{2}+4}{22}\right) \Leftrightarrow \nu \in(0,2)$.

In particular, the massless conformally coupled case corresponds to $\mu_{0}^{2}=0$ and $\xi=\frac{1}{6} \in\left(0, \frac{4}{22}\right)$, which entails $\mu^{2}=-\frac{22}{6 L^{2}}$ and, accordingly, $\nu=\frac{1}{\sqrt{3}}$.

A direct inspection of Eq. (3.8) unveils the existence of two different asymptotic behaviors at infinity. Hence, in order to select one among the infinite possible solutions at $r \rightarrow \infty$, one needs to identify a suitable criterion. This is the core of the next sections and it relies on the observation that Eq. (3.7) can be rewritten as an eigenvalue problem for a Sturm-Liouville operator, i.e.,

$$
L_{\omega^{2}} R(r)=\omega^{2} R(r),
$$

where

$$
L_{\omega^{2}}:=-\frac{1}{q(r)}\left(\frac{d}{d r}\left(p(r) \frac{d}{d r}\right)+v(r)\right),
$$

with coefficient functions:

$$
q(r)=\frac{4 L^{6} r}{\kappa L^{2}+2 r^{2}}
$$




$$
\begin{gathered}
p(r)=\kappa L^{2} r^{3}+2 r^{5}, \\
v(r)=2 L^{2} r\left(-\mu^{2} r^{2}+\lambda\right) .
\end{gathered}
$$

For later convenience, observe that the Sturm-Liouville operator satisfy the following properties under conjugation and reflection of $\omega \in \mathbb{C}$ :

$$
\begin{gathered}
L_{\bar{\omega}^{2}}=\overline{L_{\omega^{2}}}, \\
L_{(-\omega)^{2}}=L_{(\omega)^{2}} .
\end{gathered}
$$

\section{THE RADIAL EQUATION ON LIFSHITZ SPACETIME}

In this section, we study the solutions of the radial equation (3.7) on $\mathrm{Lif}_{0}$ using the tools proper of SturmLiouville theory, see e.g., [27]. First, we show that these solutions can be constructed starting from an associated confluent hypergeometric equation. Subsequently, in Sec. IV B we choose a convenient basis of solutions and, in Sec. IV C, we analyze whether they lie in a suitably specified Hilbert space. This analysis will highlight whether at radial infinity we can impose generalized Robin boundary conditions, as detailed in Sec. IV E. As a by product, in Sec. IV F we construct the radial Green function. Finally, in Sec. IV G, we study the existence of bound states, which is tantamount to determining which among the admissible boundary conditions yield physically sensible two-point functions. We emphasize that a construction and an analysis similar to the following one has been already applied for a scalar field on an anti-de Sitter spacetime in $[10,12]$, on a rotating BTZ black hole spacetime in [28] and on a massless topological black hole in [15]. In particular, here we follow the same nomenclature and strategy outlined in [10] to which we refer for further details.

\section{A. The radial equation as a confluent hypergeometric equation}

If one considers the coordinate change

$$
r \mapsto u=\frac{i L^{3} \omega}{r^{2}} \in(0, \infty),
$$

the radial equation (3.7) for $\kappa=0$ reads

$u R^{\prime \prime}(u)-R^{\prime}(u)-\frac{1}{4}\left(\frac{L^{2} \mu^{2}}{u}+\frac{i \lambda}{L \omega}+u\right) R(u)=0$.

At radial infinity, which is now located at $u=0, R(u) \sim$ $u^{1 \pm \frac{\nu}{2}}$ in agreement with Eq. (3.8). At $u=\infty$, we find that $R(u) \sim e^{ \pm u / 2}$. Thus, we make the ansatz

$$
R(u)=e^{-u / 2}\left(\frac{u}{i L^{3} \omega}\right)^{\frac{1}{2}(2+\nu)} w(u) .
$$

It follows that this is a solution of Eq. (4.2) if and only if $w(u)$ is a solution of the confluent hypergeometric equation

$$
u w^{\prime \prime}(u)+\left(b_{0}-u\right) w^{\prime}(u)-a_{0} w(u)=0,
$$

with

$$
\begin{aligned}
& a_{0}=\frac{1+\nu}{2}+\frac{i \lambda}{4 L \omega}, \\
& b_{0}=1+\nu .
\end{aligned}
$$

\section{B. The radial solutions}

In view of the results of the previous section, a basis $\left\{R_{1\left(u_{0}\right)}(u), R_{2\left(u_{0}\right)}(u)\right\}$ of solutions of the radial equation near a point $u_{0}$ can be written in terms of a basis $\left\{w_{1\left(u_{0}\right)}(u), w_{2\left(u_{0}\right)}(u)\right\}$ of Eq. (4.4). That is, for $j \in\{1,2\}$ we define

$$
R_{j\left(u_{0}\right)}(u)=e^{-u / 2}\left(\frac{u}{i L^{3} \omega}\right)^{\frac{1}{2}(2+\nu)} w_{j\left(u_{0}\right)}(u) .
$$

In particular we are interested in the case $u_{0}=0$ or $u_{0} \rightarrow \infty$. When $b_{0} \notin \mathbb{Z}$, satisfactory solutions of (4.4) near the endpoint $u_{0}$ are given in Table I. Moreover, a solution written in terms of a basis chosen at one endpoint can be written as a linear combination of the solutions chosen to form a basis at the other endpoint. This linear combination is called a "fundamental relation", or a "connection formula". In Sec. IV F, we make use of the fundamental relation connecting $R_{1(0)}(r)$ with the solutions at radial infinity, namely

TABLE I. Chosen bases of the confluent hypergeometric equation (4.4).

\begin{tabular}{lll}
\hline \hline Endpoint & & \multicolumn{1}{c}{ Basis } \\
\hline$u=0$ & $r=\infty$ & $w_{1(0)}(u)=M\left(a_{0}, b_{0} ; u\right)$ \\
& & $w_{2(0)}(u)=\left(i L^{3} \omega\right)^{\nu} u^{1-b_{0}} M\left(a_{0}-b_{0}+1,2-b_{0} ; u\right)$ \\
& & $w_{1(\infty)}(u)=U\left(a_{0}, b_{0} ; u\right)$ \\
& & $w_{2(\infty)}(u)=e^{u} U\left(b_{0}-a_{0}, b_{0} ;-u\right)$ \\
\hline \hline
\end{tabular}




$$
R_{1(0)}(r)=A_{0} R_{1(\infty)}(r)+B_{0} R_{2(\infty)}(r),
$$

with coefficients

$$
\begin{aligned}
A_{0} & :=\frac{\Gamma\left(1-b_{0}\right)}{\Gamma\left(a_{0}-b_{0}+1\right)}, \\
B_{0} & :=\frac{\Gamma\left(b_{0}-1\right)}{\Gamma\left(a_{0}\right)}\left(i L^{3} \omega\right)^{-\nu} .
\end{aligned}
$$

The confluent hypergeometric functions $M$ and $U$, which are also known as Kummer's functions, are discussed in [29], Ch. 13. As detailed in Sec. 13.2 of [29], Ch. 13, when the parameter $b_{0}$ assumes integer values, and depending on the value of the parameter $a_{0}$, we have to replace the solution $w_{2(0)}$ as stated in Table I. In this case, logarithmic corrections have to be taken into account, as it happens in [30], and the new solution does not necessarily abide to a square-integrability condition as discussed in the next section. For this reason we avoid writing these solutions explicitly, and we refer the reader to [29], Ch. 13, for their expressions. Specifically, we focus on effective masses for the scalar field that allow for boundary conditions besides the Dirichlet one. This restricts the parameter $\nu$ to the interval $(0,1)$ and it selects the radial solutions as given in Table I.

\section{Square-integrability conditions}

Following the rationale of Sturm-Liouville theory-see in particular [10,27]—we are looking for solutions $R(r)$ of the radial equation such that there exists a neighborhood of $r_{0}$ for which $R(r)$ is square integrable with respect to the measure $q(r)$ as defined in Eq. (3.10c). Next in order, we verify such property for the radial solutions (4.5), written in terms of the solutions of Table I, hence classifying the endpoints of the domain of the radial equation with $\kappa=0$ into limit point or limit circle, according to Weyl's endpoint classification, see [27].

At $u=0(r=\infty)$, the solution $w_{1(0)}(u)$ reduces to 1 , while $w_{2(0)}(u)$ tends asymptotically to $u^{1-b_{0}}$. Therefore, the radial solutions (4.5), written in terms of $r$ instead of $u$, behave asymptotically as:

$$
\begin{aligned}
& R_{1(\infty)}(r) \sim r^{-2-\nu}, \\
& R_{2(\infty)}(r) \sim r^{-2+\nu} .
\end{aligned}
$$

Taking into account the measure (3.10c), one finds that both solutions lay in $L^{2}\left(\left(r_{0}, \infty\right) ; q(r)\right), \forall r_{0}>0$, if and only if $\nu \in(0,1) \cup(1,2)$. For this range of values of $\nu$, $r=\infty$ is a limit circle. For $\nu=1$ or $\nu \geq 2$, only $R_{1(0)}(r)$ is square integrable and $r=\infty$ is a limit point.
On the other hand, the endpoint $r=0$ is always a limit point. By direct inspection, it follows that:

$$
\begin{aligned}
\left|R_{1(0)}(r)\right|^{2} & \sim e^{+\frac{L^{3}}{r^{2}} \operatorname{Im}(\omega)}, \\
\left|R_{2(0)}(r)\right|^{2} & \sim e^{-\frac{L^{3}}{r^{2}} \operatorname{Im}(\omega)} .
\end{aligned}
$$

Therefore, the only solution lying in $L^{2}\left(\left(0, r_{0}\right) ; q(r)\right)$ for all $r_{0}<\infty$ is

$$
R_{0}(r)= \begin{cases}R_{1(0)}(r), & \text { for } \operatorname{Im}(\omega)<0 \\ R_{2(0)}(r), & \text { for } \operatorname{Im}(\omega)>0\end{cases}
$$

\section{Symmetries under conjugation and reflection}

In this section, we show that the properties of the SturmLiouville operator as per Eq. (3.11a) reflect themselves in those of the solutions (4.5) of the radial equation. First, let us make the $\omega$-dependence explicit, by denoting the parameter $a_{0}$ by $a_{0}(\omega)$ and defining the auxiliary function:

$$
\alpha(r, \omega)=e^{-\frac{i L^{3} \omega}{2 r^{2}}} r^{-2-\nu} .
$$

The basis at the endpoint $r=\infty$ reads:

$$
\begin{gathered}
R_{1(\infty)}(r, \omega)=\alpha(r, \omega) M\left(a_{0}(\omega), b_{0} ; i L^{3} \omega r^{-2}\right), \quad(4.11 \mathrm{a}) \\
R_{2(\infty)}(r, \omega)=\alpha(r, \omega) r^{2 \nu} M\left(a_{0}(\omega)-b_{0}+1,2-b_{0} ; i L^{3} \omega r^{-2}\right),
\end{gathered}
$$

and at $r=0$ :

$$
\begin{gathered}
R_{1(0)}(r, \omega)=\alpha(r, \omega) U\left(a_{0}(\omega), b_{0} ; i L^{3} \omega r^{-2}\right), \\
R_{2(0)}(r, \omega)=\alpha(r, \omega) e^{\frac{i L^{3} \omega}{r^{2}}} U\left(b_{0}-a_{0}(\omega), b_{0} ;-i L^{3} \omega r^{-2}\right) .
\end{gathered}
$$

From the definition of the parameters $a_{0}(\omega)$ and $b_{0}$, see Eq. (4.4b), it follows:

$$
\begin{gathered}
a_{0}(\bar{\omega})=\overline{b_{0}-a_{0}(\omega)}, \\
a_{0}(-\omega)=b_{0}-a_{0}(\omega) .
\end{gathered}
$$

Taking these properties into account (4.8) together with the Kummer's transformation:

$$
M\left(a_{0}(\omega), b_{0}, u\right)=e^{u} M\left(b_{0}-a_{0}(\omega), b_{0},-u\right),
$$

we obtain, for $j_{1} \in\{1,2\}$, that the basis at $r=\infty$ satisfies

$$
R_{j_{1}(\infty)}(r, \bar{\omega})=\overline{R_{j_{1}(\infty)}(r, \omega)} .
$$


Also, for $j_{2} \in\{1,2\}$, with $j_{1} \neq j_{2}$, we find that the basis at $r=0$ satisfy:

$$
R_{j_{1}(0)}(r, \bar{\omega})=\overline{R_{j_{2}(0)}(r, \omega)} .
$$

Furthermore, since the solutions depend on $\omega$ only through a factor $i \omega$, the conjugation properties imply that for frequencies such that $\omega^{2} \in \mathbb{R}$, the solutions at $r=\infty$ are real valued. At the same time, the solutions at $r=0$ are not necessarily real valued for real frequencies, but in this case one is the complex conjugate of the other.

\section{E. Robin boundary conditions at $r=\infty$}

In the range $\nu \in(0,1) \cup(1,2)$, both solutions are square integrable at radial infinity. Hence, in the spirit of SturmLiouville theory, in this case an admissible solution is a linear combination of $R_{1(\infty)}(r)$ and $R_{2(\infty)}(r)$. In fact, there exists a one-parameter family of suitable boundary conditions such that, for each $\gamma \in[0, \pi)$, the solution

$$
R_{\gamma}(r)=\cos (\gamma) R_{1(\infty)}(r)+\sin (\gamma) R_{2(\infty)}(r)
$$

satisfies

$$
\begin{aligned}
& \lim _{r \rightarrow \infty}\left(\cos (\gamma) W_{r}\left[R_{\gamma}(r), R_{1(\infty)}(r)\right]\right. \\
& \left.\quad+\sin (\gamma) W_{r}\left[R_{\gamma}(r), R_{2(\infty)}(r)\right]\right)=0,
\end{aligned}
$$

where we recall that, given two differentiable functions $f_{1}(x)$ and $f_{2}(x)$ defined on an interval $I \subseteq \mathbb{R}$, the Wronskian $W$ reads

$W_{x}\left[f_{1}(x), f_{2}(x)\right]=f_{1}(x) \frac{d f_{2}(x)}{d x}-\frac{d f_{1}(x)}{d x} f_{2}(x)$.

For $\gamma=0$ we recover Dirichlet boundary condition, given that $R_{\gamma}(r)$ reduces to the principal solution $R_{1(\infty)}$. For $\gamma=\frac{\pi}{2}$ we have Neumann boundary condition, for a fixed secondary solution $R_{2(\infty)}$. The cases $\gamma \in\left(0, \frac{\pi}{2}\right) \cup\left(\frac{\pi}{2}, \pi\right)$, are referred to as Robin boundary conditions. In reality, Dirichlet and Neumann can be seen as particular cases of Robin boundary conditions, but here we make the distinction for convenience in future referencing.

Given that the basis chosen at $r=\infty$ satisfy properties (4.15), it holds:

$$
R_{\gamma}(r, \bar{\omega})=\overline{R_{\gamma}(r, \omega)} .
$$

\section{F. The radial Green function}

Using the results of the previous sections we can construct the Green's distribution $\mathcal{G}_{\omega}\left(r, r^{\prime}\right)$ of the differential operator $L_{\omega^{2}}$, given by Eq. (3.10b), i.e., a solution of

$$
\begin{aligned}
\left(\left(L_{\omega^{2}}-\omega^{2}\right) \otimes \mathbb{1}\right) \mathcal{G}_{\omega}\left(r, r^{\prime}\right) & =\left(\mathbb{1} \otimes\left(L_{\omega^{2}}-\omega^{2}\right)\right) \mathcal{G}_{\omega}\left(r, r^{\prime}\right) \\
& =\frac{\delta\left(r-r^{\prime}\right)}{q(r)} .
\end{aligned}
$$

Consider the range of values $\nu \in(0,1) \cup(1,2)$. Following [31], we are able to write $\mathcal{G}_{\omega}\left(r, r^{\prime}\right)$ in terms of solutions of the radial solutions $R_{0}$ and $R_{\gamma}$, as in (4.9) and (4.17), as

$$
\begin{aligned}
\mathcal{G}_{\omega}\left(r, r^{\prime}\right)= & \frac{1}{\mathcal{N}_{\omega}}\left(\Theta\left(r^{\prime}-r\right) R_{0}(r) R_{\gamma}\left(r^{\prime}\right)\right. \\
& \left.+\Theta\left(r-r^{\prime}\right) R_{0}\left(r^{\prime}\right) R_{\gamma}(r)\right) .
\end{aligned}
$$

For $p(r)$ as in Eq. (3.10d), the normalization $\mathcal{N}_{\omega}$ is

$$
\mathcal{N}_{\omega}:=-p(r) W_{r}\left[R_{0}(r), R_{\gamma}(r)\right] .
$$

The solution $R_{0}$ is given by Eq. (4.9), while $R_{\gamma}$ is given by Eq. (4.17) together with Eqution (4.7). Assuming $\operatorname{Im}(\omega)<0$, we have $R_{0}(r)=R_{1(0)}(r)$. Considering the fundamental relation that connects the solution $R_{1(0)}(r)$ with the solutions at radial infinity given by Eq. (4.6) and taking into account the Wronskian [29](13.2.33),

$$
W_{u}\left[w_{1(0)}, w_{2(0)}\right]=\left(i L^{3} \omega\right)^{\nu}\left(1-b_{0}\right) u^{-b_{0}} e^{u},
$$

the normalization $(4.22 b)$ reads

$\mathcal{N}_{\omega}=4 \nu\left\{B_{0} \cos (\gamma)-A_{0} \sin (\gamma)\right\}, \quad$ for $\operatorname{Im} \omega<0$.

With respect to frequencies $\bar{\omega}$ with $\operatorname{Im}(\bar{\omega})>0$, we take $R_{0}(r)=R_{2(0)}(r)$ and the normalization in the upper part of the complex plane, by an analogous computation and making the frequency dependence of $a_{0}$ explicit, reads

$$
\begin{aligned}
\mathcal{N}_{\bar{\omega}}= & 4 \nu\left\{\left(-i L^{3} \bar{\omega}\right)^{-\nu} \cos (\gamma) \frac{\Gamma\left(b_{0}-1\right)}{\Gamma\left(b_{0}-a_{0}(\bar{\omega})\right)}\right. \\
& \left.-\sin (\gamma) \frac{\Gamma\left(1-b_{0}\right)}{\Gamma\left(1-a_{0}(\bar{\omega})\right)}\right\}, \quad \text { for } \operatorname{Im} \bar{\omega}>0 .
\end{aligned}
$$

The relations individuated in Eq. (4.8) imply that, with respect to complex conjugation, the normalization and the radial Green function satisfy

$$
\mathcal{N}_{\bar{\omega}}=\overline{\mathcal{N}}_{\omega} \quad \text { and } \quad \mathcal{G}_{\bar{\omega}}=\overline{\mathcal{G}_{\omega}} .
$$

\section{G. On the existence of bound states}

We can limit ourselves to studying in detail either the case $\operatorname{Im}(\omega)<0$ or $\operatorname{Im}(\omega)>0$, and then, by symmetry, we extend the results to the other one. Next, we compute the normalization and we verify the existence of bound states, assuming $\operatorname{Im}(\omega)<0$. The poles of the radial Green function are the zeros of the normalization constant. In the following, we state the conditions that yield $\mathcal{N}_{\omega}=0$ for 
Dirichlet, Neumann and Robin boundary conditions. We recall that we are considering $\nu \in(0,1) \cup(1,2), n \in \mathbb{N}_{0}$ and that the Gamma function satisfies

$$
\frac{1}{\Gamma(z)}=0 \Leftrightarrow z \in \mathbb{Z}_{0}^{-} .
$$

\section{Dirichlet boundary condition, $\gamma=0$}

$\mathcal{N}_{\omega}=0 \Leftrightarrow a_{0}=-n \Leftrightarrow \omega=-i \frac{\lambda}{2 L(1+\nu+2 n)}$.

The frequencies above have $\operatorname{Im}(\omega)>0$, because $\lambda \leq 0$, and can thus be neglected.

\section{Neumann boundary condition, $\gamma=\pi / 2$}

$\mathcal{N}_{\omega}=0 \Leftrightarrow a_{0}-b_{0}+1=-n \Leftrightarrow \omega=-i \frac{\lambda}{2 L(1-\nu+2 n)}$.

For $\nu \in(0,1), 1-\nu+2 n>0$ and the frequencies above have positive imaginary part. At the same time, for $\nu \in$ $(1,2)$ and $n=0$, the denominator is negative, and for each $\lambda$ there is a unique pole with negative imaginary part at

$$
\omega_{p}=-i \frac{\lambda}{2 L(1-\nu)} .
$$

3. Robin boundary conditions, $\gamma \in(\mathbf{0}, \pi / 2) \cup(\pi / 2, \pi)$

Define

$$
\begin{aligned}
& \chi(\omega):=\tan (\gamma)-\Xi(\omega), \\
& \Xi(\omega):=\left(i L^{3} \omega\right)^{-\nu} \frac{\Gamma\left(a_{0}-b_{0}+1\right)}{\Gamma\left(a_{0}\right)} \frac{\Gamma\left(b_{0}-1\right)}{\Gamma\left(1-b_{0}\right)} .
\end{aligned}
$$

The zeros of the normalization are solutions of

$$
\chi(\omega)=0 .
$$

The zeros and poles of $\Xi(\omega)$ are, respectively, the zeros of the normalization for Dirichlet and Neumann boundary conditions. Thus its zeros all lie in the upper half on the $\omega$ complex plane and it has no poles for $\nu \in(0,1)$, and one pole per $\lambda$ for $\nu \in(1,2)$.

Although Eq. (4.32) is transcendental, it always admits a solution whenever $\Xi(\omega)$ is real valued. In addition, the set of frequencies that solves Eq. (4.32) lies in the point spectrum of the differential operator determined by the radial part of the Klein-Gordon operator. Since we consider solely self-adjoint extensions, its $\omega^{2}$-spectrum is necessarily within $\mathbb{R}$. Therefore, we conclude that $\mathcal{N}_{\omega}=0 \Leftrightarrow \omega=-i|\operatorname{Im}(\omega)|$, as illustrated in Fig. 2 . Observe that the argument above does not apply for

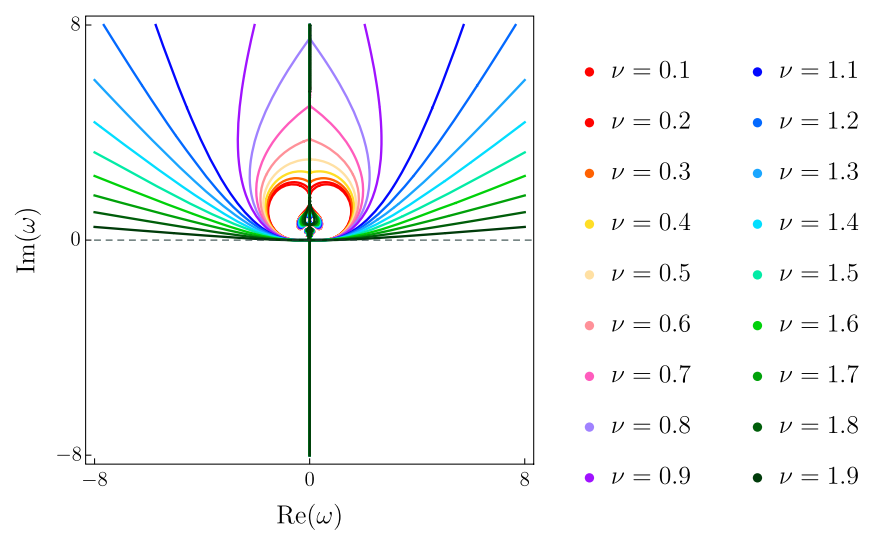

FIG. 2. Contour lines of $\operatorname{Im}(\Xi)=0$ for $L=1, \lambda=-3$ and several values of $\nu$. For $\operatorname{Im}(\omega)<0$, only $\omega=i \operatorname{Im}(\omega)$ is present.

$\operatorname{Im}(\omega)>0$, since the upper part of the $\omega$-complex plane is not in the domain of $\mathcal{N}_{\omega}$, and in fact, we notice that it has more lines of real-phase for frequencies with positive imaginary part. Next, we analyze the behavior of $\Xi$ along the negative imaginary axis, i.e., for $\omega=i \operatorname{Im}(\omega)$ with $\operatorname{Im}(\omega)<0$, for $\nu \in(0,1)$ and $\nu \in(1,2)$, separately. Observe that

$$
\begin{aligned}
\lim _{\omega \rightarrow 0} \Xi(i \omega) & =\left(\frac{-\lambda L^{2}}{4}\right)^{-\nu} \frac{\Gamma(\nu)}{\Gamma(-\nu)}, \\
\lim _{\omega \rightarrow \omega_{p}} \Xi(\omega) & =\infty, \\
\lim _{\omega \rightarrow \infty} \Xi(-i \omega) & =0,
\end{aligned}
$$

and note that the right hand side of Eq. (4.23a) is negative if $\nu \in(0,1)$, and positive if $\nu \in(1,2)$.

a. The analysis for $\nu \in(0,1)$ : The behavior of $\Xi$ for general parameters, with $\nu \in(0,1)$, is illustrated in Fig. 3. It has no poles, and as $\omega$ goes to zero, $\Xi$ goes to a $\lambda$-dependent limit (4.33a). When $\Xi(\omega)$ lies in the interval $I_{1}, \chi(\omega)$ has one simple zero. When $\Xi(\omega)$ lies in the interval $I_{2}, \chi(\omega)$ has no zeros. This means that for each $\lambda$-mode, there is a different critical interval of Robin boundary conditions for which the radial Green function has poles, given by $\gamma \in\left[\gamma_{c}^{\lambda}, \pi\right)$ with:

$$
\gamma_{c}^{\lambda}:=\arctan \left(\left(\frac{-\lambda L^{2}}{4}\right)^{-\nu} \frac{\Gamma(\nu)}{\Gamma(-\nu)}\right)
$$

However, we have that $\gamma_{c}^{\lambda} \in(\pi / 2, \pi)$, which means that for all $\lambda$-modes, there are no bound states for $\gamma \in[0, \pi / 2]$.

b. The analysis for $\nu \in(1,2)$ : The behavior of $\Xi$ for general parameters, with $\nu \in(1,2)$, is illustrated in Fig. 4. In this case, $\Xi$ has one pole for each $\lambda$-mode. $\chi(\omega)$ has a simple zero when $\Xi$ lies in $I_{1}$, and no zeros when it lies in $I_{2}$. In this 


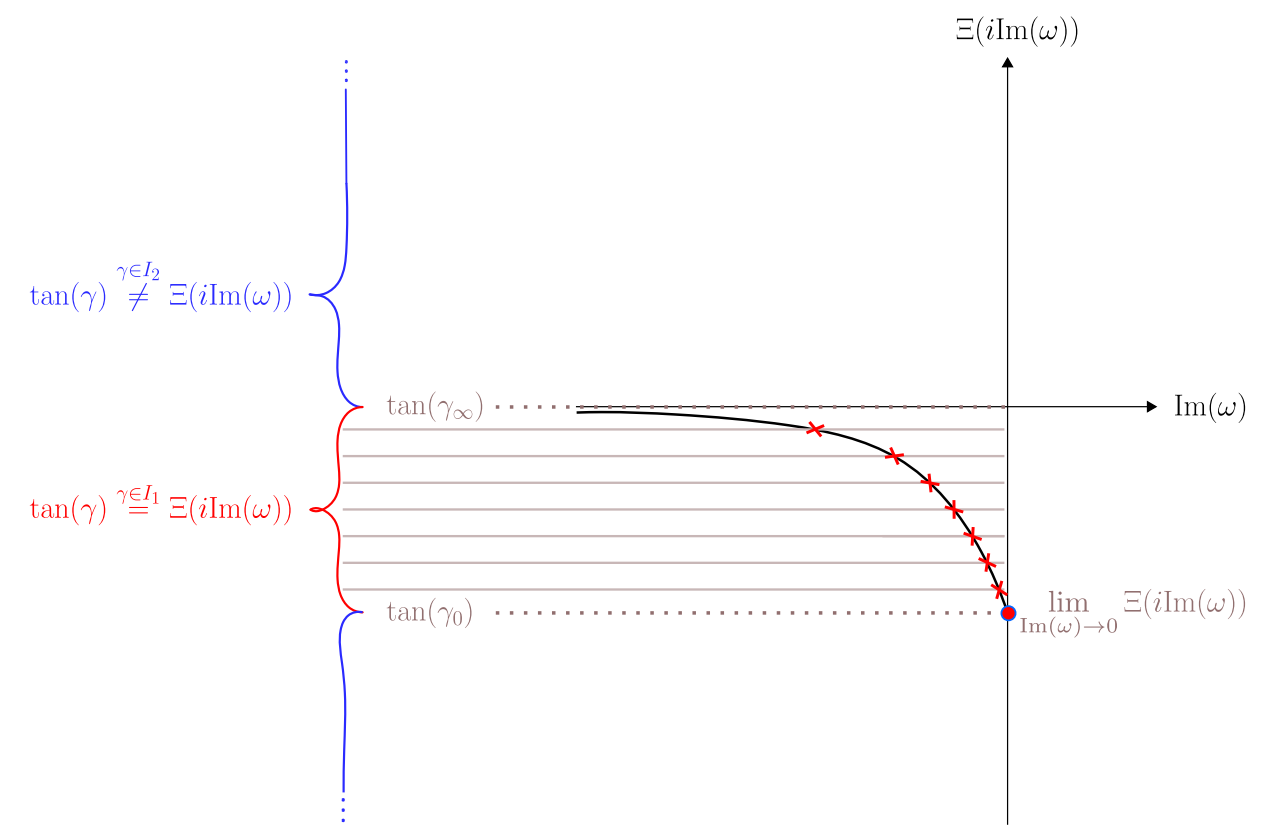

FIG. 3. The behavior of $\Xi$ for purely imaginary frequencies with $\operatorname{Im}(\omega)<0$ for $\nu \in(0,1)$.

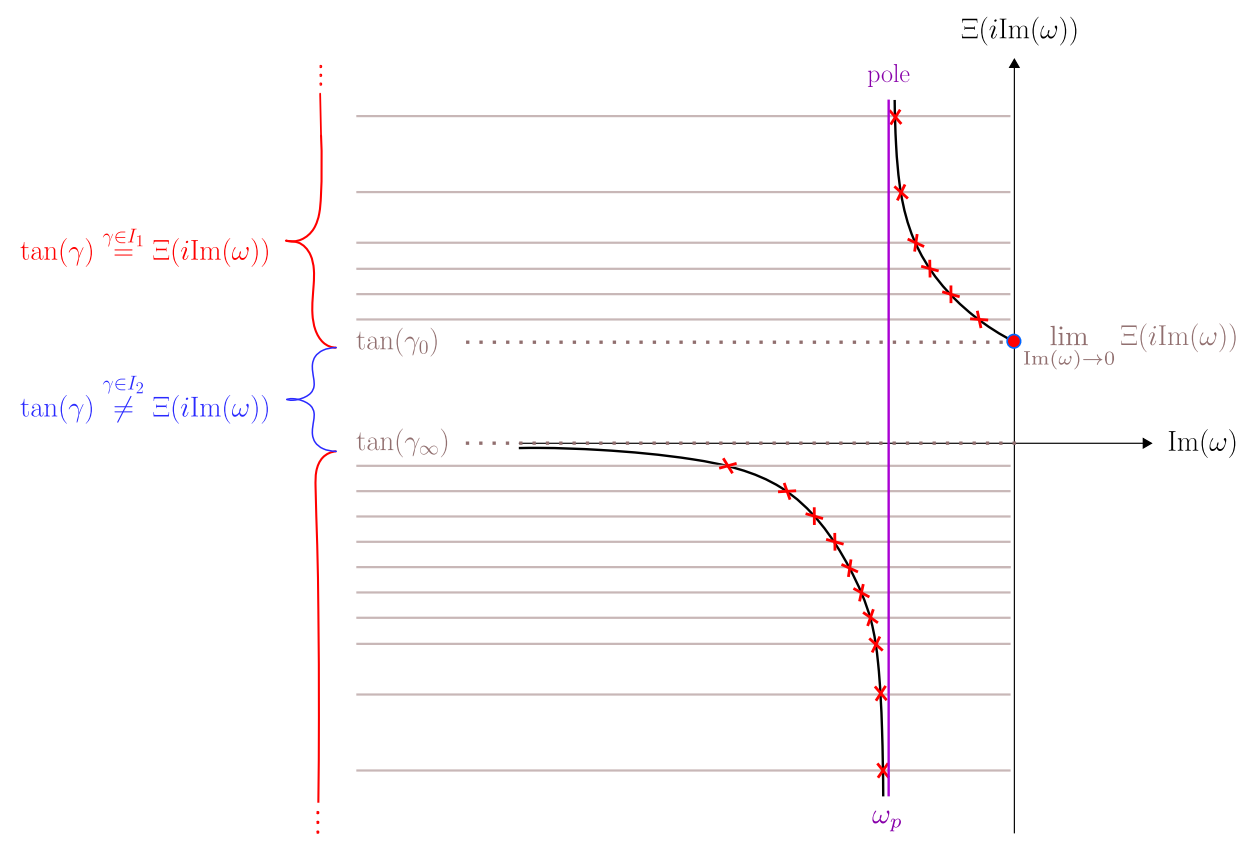

FIG. 4. The behavior of $\Xi$ for purely imaginary frequencies with $\operatorname{Im}(\omega)<0$ for $\nu \in(1,2)$.

case, $I_{2}$ corresponds to boundary conditions $\gamma \in\left(0, \gamma_{c}^{\lambda}\right)$. In short, for each $\lambda$-mode, the Robin boundary conditions for which there are no bound states are:

$\gamma \in\left(0, \gamma_{c}^{\lambda}\right) \quad$ with $\quad \gamma_{c}^{\lambda} \in \begin{cases}(\pi / 2, \pi) & \nu \in(0,1) \\ {[0, \pi / 2)} & \nu \in(1,2)\end{cases}$

We conclude that, if $\nu \in(0,1)$, then, for all $\lambda$-modes, the boundary conditions for which there are no bound states are $\gamma \in[0, \pi / 2]$. At the same time, if $\nu \in(1,2)$, for all boundary conditions $\gamma \in(0, \pi)$ there are $\lambda$-modes for which there are bound states, but Dirichlet and mode-dependent boundary conditions can be suitably imposed.

\section{THE RADIAL EQUATION ON THE HYPERBOLIC LIFSHITZ BLACK HOLE}

In this section, we study the Klein-Gordon equation on Lif $_{-1}$, an hyperbolic Lifshitz black hole. We follow the 
same procedure as in Sec. IV. First, in Sec. VA, we show that the radial equation (3.7) can be reduced to a hypergeometric equation under a suitable coordinate transform. In Sec. V B, we individuate the bases of radial solutions that are numerically satisfactory near each singularity. Then, in Sec. V C, we study the conditions for the solutions to be square integrable with respect to the measure (3.10c). With the symmetry properties described in Sec. VD, we study the radial Green function defined in Sec. V E. Finally, in Sec. VF, we verify for which boundary conditions the radial Green function has poles.

\section{A. The radial equation as a hypergeometric equation}

Applying the coordinate change

$$
r \mapsto s=\frac{2 r^{2}-L^{2}}{2 r^{2}} \in(0,1),
$$

and making the ansatz

$$
R(s)=s^{-i L \omega}(1-s)^{\frac{1}{2}(2+\nu)} h(s),
$$

we obtain that $R(s)$ solves the radial equation if and only if $h(s)$ is a solution of the hypergeometric equation

$$
s(1-s) h^{\prime \prime}(s)+(c-(a+b+1) s) h^{\prime}(s)-a b h(s)=0,
$$

with parameters

$$
\begin{gathered}
a=\frac{1+\nu}{2}-i L \omega-\Upsilon, \\
b=\frac{1+\nu}{2}-i L \omega+\Upsilon, \\
c=1-2 i L \omega,
\end{gathered}
$$

where

$$
\Upsilon=-\frac{\sqrt{1+2 \lambda-4 L^{2} \omega^{2}}}{2} .
$$

\section{B. The radial solutions}

When the hypergeometric parameters

$$
c, c-a-b, \text { and } a-b
$$

are not integers, numerically satisfactory bases of solutions of the hypergeometric equation (5.3a), dubbed

$$
\left\{h_{1\left(s_{0}\right)(s)}, h_{2\left(s_{0}\right)}(s)\right\}
$$

at each singular endpoint $s_{0} \in\{0,1\}$, are given in Table II. They are, respectively, given by expressions (15.10.11)(15.10.14) in [29], Ch. 15.

A basis at $s=s_{0}$ for the radial equation, dubbed $\left\{R_{1\left(s_{0}\right)}(s), R_{2\left(s_{0}\right)}(s)\right\}$, descends from the ansatz (5.2); for $j \in\{1,2\}$

$$
R_{j\left(s_{0}\right)}(s)=s^{-i L \omega}(1-s)^{\frac{1}{2}(2+\nu)} h_{j\left(s_{0}\right)}(s) .
$$

For the discussion in Sec. VE, we state the fundamental relation connecting the square-integrable solution at $r=$ $L / \sqrt{2}$ with the solutions at radial infinity. This is given by [29] (15.10.22)

$$
R_{2(L / \sqrt{2})}(r)=A_{-1} R_{1(\infty)}(r)+B_{-1} R_{2(\infty)}(r),
$$

with coefficients

$$
A_{-1}:=\frac{\Gamma(2-c) \Gamma(c-a-b)}{\Gamma(1-a) \Gamma(1-b)},
$$

$$
B_{-1}:=\frac{\Gamma(2-c) \Gamma(a+b-c)}{\Gamma(a-c+1) \Gamma(b-c+1)} .
$$

\section{Square-integrability conditions}

Analogously to Sec. IV C, we are interested at unveiling whether the solutions of the radial equation are square integrable close to the endpoints $r=\frac{L}{\sqrt{2}}$ and $r \rightarrow \infty$ with respect to the measure $q(r) d r$ where $q$ is defined in Eq. (3.10c). At the endpoint $s=0, r=L / \sqrt{2}$, the radial solutions (5.2), behave as:

$$
\left|R_{1(L / \sqrt{2})}(r)\right|^{2} \sim\left(2 r^{2}-L^{2}\right)^{+2 L \operatorname{Im}(\omega)},
$$

TABLE II. Chosen bases of the hypergeometric equation (5.3a).

\begin{tabular}{lll}
\hline \hline Endpoint & & \multicolumn{1}{c}{ Basis } \\
\hline$s=0$ & $r=\frac{L}{\sqrt{2}}$ & $h_{1(0)}(s)=F(a, b ; c ; s)$ \\
& & $h_{2(0)}(s)=s^{1-c} F(a-c+1, b-c+1 ; 2-c ; s)$ \\
$s=1$ & $h_{1(1)}(s)=F(a, b ; a+b+1-c ; 1-s)$ \\
& & $h_{2(1)}(s)=(1-s)^{c-a-b} F(c-a, c-b ; c-a-b+1 ; 1-s)$ \\
\hline \hline
\end{tabular}




$$
\left|R_{2(L / \sqrt{2})}(r)\right|^{2} \sim\left(2 r^{2}-L^{2}\right)^{-2 L \operatorname{Im}(\omega)} .
$$

With respect to the measure $(3.10 \mathrm{c})$, we obtain that $R_{1(L / \sqrt{2})}(r)$ is square integrable if and only if $\operatorname{Im}(\omega)>0$, and $R_{2(L / \sqrt{2})}(r)$, if $\operatorname{Im}(\omega)<0$. Thus $r=L / \sqrt{2}$ is limit point and no boundary condition is necessary.

At the endpoint $s=1, r \rightarrow \infty$, the asymptotic behavior of the solutions is consistent with Eq. (3.8a):

$$
\begin{gathered}
\left|R_{1(\infty)}(r)\right|^{2} \sim r^{-4-2 \nu}, \\
\left|R_{2(\infty)}(r)\right|^{2} \sim r^{-4+2 \nu} .
\end{gathered}
$$

Identically to the case $\kappa=0$ it descends that, for $\nu \in$ $(0,1) \cup(1,2)$ both solutions are square integrable and $r=\infty$ is a limit circle. For $\nu=1$ or $\nu \geq 2$, only $R_{1(\infty)}(r)$ is square integrable and radial infinity is limit point.

With the analyses above in mind, we denote the most general square-integrable radial solutions that are suitable at the singular endpoint $r=L / \sqrt{2}$ and $r=\infty$ by $R_{L / \sqrt{2}}(r)$ and $R_{\gamma}(r)$, respectively. Considering the solutions (5.4) as functions of $r$ instead of $s$, they are given by

$$
\begin{aligned}
& R_{L / \sqrt{2}}(r):= \begin{cases}R_{1(L / \sqrt{2})}(r), & \text { if } \operatorname{Im}(\omega)>0, \\
R_{2(L / \sqrt{2})}(r), & \text { if } \operatorname{Im}(\omega)<0,\end{cases} \\
& R_{\gamma}(r):=\cos (\gamma) R_{1(\infty)}(r)+\sin (\gamma) R_{2(\infty)}(r) .
\end{aligned}
$$

The solution $R_{\gamma}$ satisfies the generalized Robin boundary conditions at radial infinity parametrized by $\gamma \in[0, \pi)$, just as described in detail in Sec. IV E for the case $k=0$, but with $R_{1(\infty)}(r)$ and $R_{1(\infty)}(r)$ built out of (5.4).

\section{Symmetries under conjugation and reflection}

The radial solutions for $\kappa=-1$ satisfy properties analogous to the ones described in Sec. IV D. The parameters of the hypergeometric equation are such that

$$
\begin{gathered}
a(\bar{\omega})=\overline{a(\omega)-c(\omega)+1}, \\
b(\bar{\omega})=\overline{b(\omega)-c(\omega)+1}, \\
c(\bar{\omega})=\overline{2-c(\omega)} .
\end{gathered}
$$

Using these properties, we find that for $j_{1}, j_{2} \in\{1,2\}$, the radial solutions (5.4) satisfy

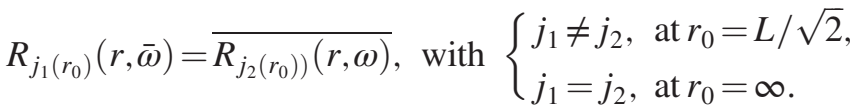

Therefore, the radial solutions (5.8) satisfy:

$$
\begin{aligned}
R_{L / \sqrt{2}}(r, \bar{\omega}) & =\overline{R_{L / \sqrt{2}}(r, \omega)}, \\
R_{\gamma}(r, \bar{\omega}) & =\overline{R_{\gamma}(r, \omega)} .
\end{aligned}
$$

\section{E. The radial Green function}

Analogously to the case $\kappa=0$, described in Sec. IV F, we define the radial Green function $\mathcal{G}_{\omega}\left(r, r^{\prime}\right)$ for $\kappa=-1$, with radial solutions given as in Eq. (5.8). Considering the range of values $\nu \in(0,1) \cup(1,2)$ and $p(r)$ as in Eq. (3.10d), the radial Green function reads

$$
\begin{aligned}
\mathcal{G}_{\omega}\left(r, r^{\prime}\right)= & \frac{1}{\mathcal{N}_{\omega}}\left(\Theta\left(r^{\prime}-r\right) R_{L / \sqrt{2}}(r) R_{\gamma}\left(r^{\prime}\right)\right. \\
& \left.+\Theta\left(r-r^{\prime}\right) R_{L / \sqrt{2}}\left(r^{\prime}\right) R_{\gamma}(r)\right),
\end{aligned}
$$

with normalization $\mathcal{N}_{\omega}$

$$
\mathcal{N}_{\omega}:=-p(r) W_{r}\left[R_{L / \sqrt{2}}(r), R_{\gamma}(r)\right]
$$

Recall that $R_{L / \sqrt{2}}$ is defined by parts with respect to the sign of the imaginary part of $\omega$. For $\operatorname{Im}(\omega)<0$, using the fundamental relation given by Eq. (5.5) and the Wronskian [29] (15.10.3), the normalization (5.12b) can be written as

$\mathcal{N}_{\omega}=L^{4} \nu\left\{B_{-1} \cos (\gamma)-A_{-1} \sin (\gamma)\right\}$, for $\operatorname{Im}(\omega)<0$.

Moreover, given the properties with respect to complex conjugation, as described in Sec. V D, it holds:

$\mathcal{N}_{\bar{\omega}}=L^{4} \nu\left\{\overline{B_{-1}} \cos (\gamma)-\overline{A_{-1}} \sin (\gamma)\right\}, \quad$ for $\operatorname{Im}(\bar{\omega})>0$.

Analogously to the case $\kappa=0$ :

$$
\mathcal{N}_{\bar{\omega}}=\overline{\mathcal{N}_{\omega}} \quad \text { and } \quad \mathcal{G}_{\bar{\omega}}\left(r, r^{\prime}\right)=\overline{\mathcal{G}_{\omega}\left(r, r^{\prime}\right)},
$$

which entails that we can analyze the case $\operatorname{Im}(\omega)<0$, and extend directly the results to $\operatorname{Im}(\omega)>0$. In the next section, we assume $\operatorname{Im}(\omega)<0$ and we investigate the zeros of the normalization.

\section{F. On the existence of bound states}

In the following, we check the existence of bound states for each boundary condition by studying the zeros of the normalization $\mathcal{N}_{\omega}$. In all cases, consider $n \in \mathbb{N}_{0}$. Observe that, since we are considering $\nu \in(0,1) \cup(1,2)$, the effective mass assumes only negative values and the coupling constant is positive as indicated in Eq. (3.9). Accordingly, for $k=-1$, we have that the parameter $\lambda$, given by Eq. (3.7d), is always negative. 


\section{Dirichlet boundary condition, $\gamma=0$}

$$
\begin{aligned}
\mathcal{N}_{\omega}=0 & \Leftrightarrow(a+2 i L \omega=-n \text { or } b+2 i L \omega=-n) \\
& \Leftrightarrow \omega=\frac{i\left(-2 \lambda+\nu^{2}+2 \nu+4 n^{2}+4 \nu n+4 n\right)}{4 L(\nu+2 n+1)} .
\end{aligned}
$$

These frequencies have positive imaginary part, and the expression above for the normalization is defined for $\operatorname{Im}(\omega)<0$, hence there are no zeros in its domain.

\section{Neumann boundary condition, $\gamma=\pi / 2$}

$$
\begin{aligned}
\mathcal{N}_{\omega}=0 & \Leftrightarrow(1-a=-n \text { or } 1-b=-n) \\
& \Leftrightarrow \omega=\frac{i\left(-2 \lambda+\nu^{2}-2 \nu+4 n^{2}-4 \nu n+4 n\right)}{4 L(-\nu+2 n+1)} .
\end{aligned}
$$

For $n>0$, the frequency above lies in the upper part of the complex-plane. For $n=0$, however, its imaginary part does assume negative values. Let $\omega_{p}$ be the frequency for $n=0$ :

$$
\omega_{p}=\frac{i(-2 \lambda+\nu(\nu-2))}{4 L(1-\nu)},
$$

and let us define the auxiliary quantities:

$$
\lambda_{c}:=-\frac{\nu(2-\nu)}{2},
$$

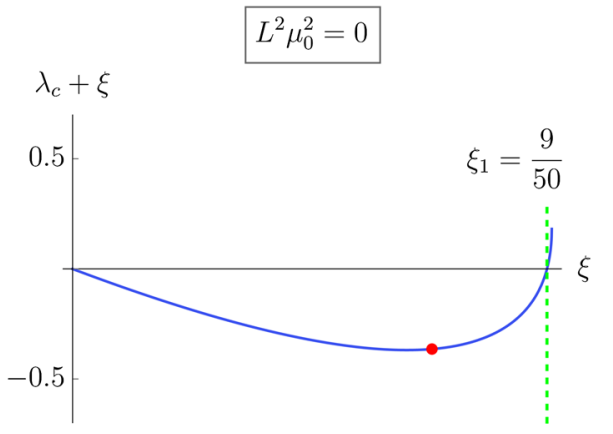

$$
L^{2} \mu_{0}^{2}=6.5
$$

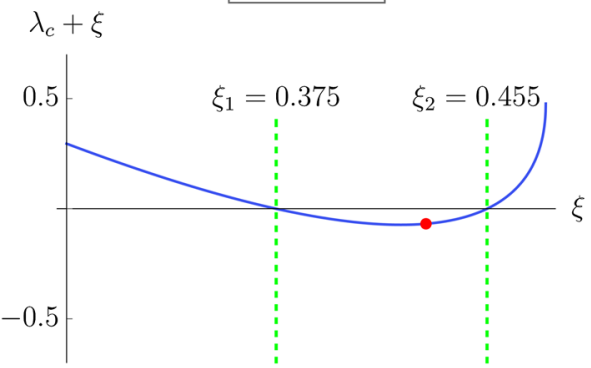

and

$$
\begin{aligned}
& \xi_{1}:=\frac{1}{100}\left(9+5 L^{2} \mu_{0}^{2}-\sqrt{81-10 L^{2} \mu_{0}^{2}}\right), \\
& \xi_{2}:=\frac{1}{100}\left(9+5 L^{2} \mu_{0}^{2}+\sqrt{81-10 L^{2} \mu_{0}^{2}}\right) .
\end{aligned}
$$

For the frequency $\omega_{p}$, we find that

$$
\operatorname{Im}\left(\omega_{p}\right)<0 \text { for } \begin{cases}\lambda>\lambda_{c}, & \text { if } \nu \in(0,1), \\ \lambda<\lambda_{c}, & \text { if } \nu \in(1,2) .\end{cases}
$$

It is easy to see that in the range $\nu \in(1,2)$, regardless of the value of $\lambda_{c}$, there is an uncountable set of $\lambda$-modes for which $\lambda<\lambda_{c}$. For $\nu \in(0,1)$, on the other hand, Eq. (5.22) is meaningful only if $\lambda_{c}<-\xi$. It follows that:

$\lambda_{c} \geq-\xi \Leftrightarrow$ either $\left\{\begin{array}{l}\text { (i) } L^{2} \mu_{0}^{2}<8.1 \text { and }\left(\xi \leq \xi_{1} \text { or } \xi \geq \xi_{2}\right) \text {; } \\ \text { (ii) } L^{2} \mu_{0}^{2} \geq 8.1 \text {. }\end{array}\right.$

For the massless field, $\xi_{1}=0$ and $\xi_{2}=\frac{9}{50}$. Therefore, $\lambda_{c} \geq-\xi$ if $\xi \in\left[\frac{9}{50}, \frac{4}{22}\right)$. For massive fields, the behavior of $\lambda_{c}$ is analogous to the one for $\mu_{0}=0$, but for increasing values of $L^{2} \mu_{0}^{2}, \lambda_{c}+\xi$ as a function of $\xi$ is shifted upwards, as illustrated in Fig. 5.

All included, the values of $L, \mu_{0}$ and $\xi$ for which no bound states emerge are:
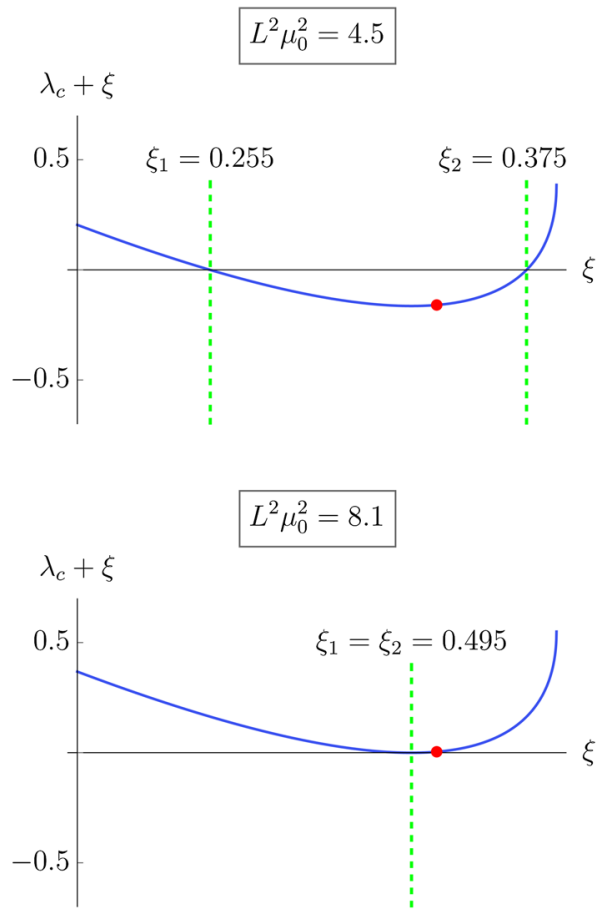

FIG. 5. $\lambda_{c}+\xi$ as a function of $\xi \in\left(\frac{L^{2} \mu_{0}^{2}}{22}, \frac{L^{2} \mu_{0}^{2}+4}{22}\right)$ for different masses. To the left of the (red) point, $\nu \in(1,2)$; to the right, $\nu \in(0,1)$. 
$\nu \in(0,1)\left\{\begin{array}{l}\text { (i) } L^{2} \mu_{0}^{2}<8.1, \xi \in\left(\xi_{1}, \xi_{2}\right) \text { and } \lambda \leq \lambda_{c} ; \\ \text { (ii) } L^{2} \mu_{0}^{2}<8.1 \text { and } \xi \notin\left(\xi_{1}, \xi_{2}\right) ; \\ \text { (iii) } L^{2} \mu_{0}^{2} \geq 8.1 ;\end{array}\right.$

$\nu \in(1,2)\left\{\begin{array}{l}\left(\text { iv) } L^{2} \mu_{0}^{2}<8.1, \xi \in\left(\xi_{1}, \xi_{2}\right) \text { and } \lambda \geq \lambda_{c} ;\right. \\ (\mathrm{v}) L^{2} \mu_{0}^{2}=8.1, \xi=\xi_{1}=\xi_{2} \text { and } \lambda=-\xi .\end{array}\right.$

Observe that for $\nu \in(0,1)$, conditions (ii) and (iii) holds for all $\lambda$-modes, while the other conditions are mode dependent.

\section{Robin boundary conditions, $\gamma \in(\mathbf{0}, \pi / 2) \cup(\pi / 2, \pi)$}

Define

$\zeta(\omega):=\tan (\gamma)-\eta(\omega)$,

$\eta(\omega):=\frac{\Gamma(a+b-c)}{\Gamma(c-a-b)} \frac{\Gamma(1-a) \Gamma(1-b)}{\Gamma(a-c+1) \Gamma(b-c+1)}$.

The bound states are the frequencies for which $\zeta(\omega)=0$, which are in turn those for which $\eta(\omega) \in \mathbb{R}$. Hence, let us study the function $\eta$. First, we note that, as in the case $\kappa=0, \eta(\omega) \in \mathbb{R} \Leftrightarrow \omega=-i|\operatorname{Im}(\omega)|$. In fact, the lines of $\operatorname{Im}(\kappa)=0$ are very similar to those of Fig. 2. Moreover,

$\lim _{\omega \rightarrow 0} \eta(\omega)=\frac{\Gamma(\nu)}{\Gamma(-\nu)} \frac{\Gamma\left(\frac{1-\nu}{2}-\frac{\sqrt{1+2 \lambda}}{2}\right) \Gamma\left(\frac{1-\nu}{2}+\frac{\sqrt{1+2 \lambda}}{2}\right)}{\Gamma\left(\frac{1+\nu}{2}+\frac{\sqrt{1+2 \lambda}}{2}\right) \Gamma\left(\frac{1+\nu}{2}-\frac{\sqrt{1+2 \lambda}}{2}\right)}$,

$\lim _{\omega \rightarrow \omega_{p}} \eta(\omega)=\infty$,

$\lim _{\omega \rightarrow \infty} \eta(-i \omega)=0$.

Note that the limit $\eta(0)$ is $\lambda$-dependent and that, whenever $\lambda$ can assume a critical value $\lambda_{c}=-\frac{\nu(2-\nu)}{2}$, then $\omega_{p}=$ 0 and $\eta(0) \rightarrow \infty$. For $\lambda \neq \lambda_{c}, \eta(0)$ is a finite number that is negative for $\left\{\lambda<\lambda_{c}\right.$ and $\left.\nu \in(0,1)\right\}$, or for $\left\{\lambda>\lambda_{c}\right.$ and $\nu \in(1,2)\}$, and positive otherwise. As a consequence the function $\eta(i \operatorname{Im}(\omega))$ with $\operatorname{Im}(\omega)<0$ behaves like the function $\Xi(i \operatorname{Im}(\omega))$, as in Fig. 3, whenever there are no bound states. Since, in this case, $\lambda$ is never equal to $\lambda_{c}$, the acceptable boundary conditions are such that

$\gamma \in\left[0, \gamma_{c}^{\lambda}\right) \quad$ with $\quad \gamma_{c}^{\lambda}:=\arctan (\eta(0)) \in\left(\frac{\pi}{2}, \pi\right)$.

Whenever there are bound states, $\eta(i \operatorname{Im}(\omega))$ behaves as $\Xi(i \operatorname{Im}(\omega))$ in Fig. 4. In this case, for each $\lambda$-mode, there are no bound states in the regime

$$
\gamma \in\left[0, \gamma_{c}^{\lambda}\right) \quad \text { with } \quad \gamma_{c}^{\lambda} \in(0, \pi / 2) .
$$

\section{THE RADIAL EQUATION ON THE SPHERICAL LIFSHITZ BLACK HOLE}

In this section, we study the Klein-Gordon equation on $\mathrm{Lif}_{1}$, a spherical Lifshitz black hole. We follow the same steps as in the previous two cases. First, in Sec. VI A, we write the radial equation as an hypergeometric equation, and, in Sec. VI B, we state the bases that are numerically satisfactory near each singular endpoint. Then, after checking, in Sec. VI C, which solutions are compatible with selfadjoint extensions of the radial part of the Klein-Gordon operator, we describe, in Sec. VID, the symmetry properties satisfied by the solutions. Finally, in Sec. VIE, we define the radial Green function and we verify, in Sec. VI F, which boundary conditions can be imposed bypassing the emergence of bound states.

Observe that, even though the notation used in the following is the same as the one of Sec. V, the parameters and functions have different definitions here.

\section{A. The radial equation as a hypergeometric equation}

Applying the coordinate change:

$$
r \mapsto s=\frac{2 r^{2}+L^{2}}{2 r^{2}} \in(1, \infty),
$$

and considering the ansatz

$$
R(s)=s^{i L \omega}(s-1)^{\frac{1}{2}(2+\nu)} h(s),
$$

we obtain that (6.2) solves the radial equation if and only if $h(s)$ is in turn a solution of the hypergeometric equation

$s(1-s) h^{\prime \prime}(s)+(c-(a+b+1) s) h^{\prime}(s)-a b h(s)=0$,

with parameters

$$
\begin{aligned}
& a=\frac{1+\nu}{2}+i L \omega-\Upsilon, \\
& b=\frac{1+\nu}{2}+i L \omega+\Upsilon, \\
& c=1+2 i L \omega,
\end{aligned}
$$

where

$$
\Upsilon=\frac{\sqrt{1-2 \lambda-4 L^{2} \omega^{2}}}{2}
$$

\section{B. The radial solutions}

A basis for the radial equation, suitable at each singular endpoint $s=s_{0} \in\{1, \infty\}$, is given by $\left\{R_{1\left(s_{0}\right)}(s), R_{2\left(s_{0}\right)}(s)\right\}$, with 
TABLE III. Chosen basis of the hypergeometric equation (6.3a).

\begin{tabular}{|c|c|c|}
\hline Endpoint & & Basis \\
\hline$s=1$ & $r=\infty$ & $\begin{array}{l}h_{1(1)}(s)=F(a, b ; a+b+1-c ; 1-s) \\
h_{2(1)}(s)=(s-1)^{c-a-b} F(c-a, c-b ; c-a-b+1 ; 1-s)\end{array}$ \\
\hline$s=\infty$ & $r=0$ & $\begin{array}{l}h_{1(\infty)}(s)=s^{-a} F(a, a-c+1 ; a-b+1 ; 1 / s) \\
h_{2(\infty)}(s)=s^{-b} F(b, b-c+1 ; b-a+1 ; 1 / s)\end{array}$ \\
\hline
\end{tabular}

$R_{j\left(s_{0}\right)}(s)=s^{i L \omega}(s-1)^{\frac{1}{2}(2+\nu)} h_{j\left(s_{0}\right)}(s)$, for $j \in\{1,2\}$,

and where $\left\{h_{1\left(s_{0}\right)(s)}, h_{2\left(s_{0}\right)}(s)\right\}$ is a basis of solutions, at $s_{0}$, of the hypergeometric equation (6.3a). When the hypergeometric parameters

$$
c, c-a-b, \quad \text { and } a-b
$$

are not integers, we consider the solutions given in Table III. Note that, since $s>1$, we have conveniently redefined the bases at $r=\infty$, in comparison with the $\kappa=0$ case that is given in Table I. The reason is that it places the branch cut of the solution $h_{2(0)}$ outside of the domain of the equation. When the above mentioned hypergeometric parameters assume integer values, another secondary solution must be chosen. As in the case $\kappa=-1$, such solutions are not square integrable. Thus, even though we consider them in the analysis, we do not include their explicit expressions here. This choice of basis grants us analogous symmetry properties with respect to complex conjugation, as we describe in the following Sec. VID.

Analogously to the previous cases, for the construction of the radial Green function in Sec. VID, we make use of the fundamental relation connecting the solution $R_{2(0)}(r)$ with those at radial infinity, given by [29] (15.10.24)

$$
R_{2(0)}(r)=A_{+1} R_{1(\infty)}(r)+B_{+1} R_{2(\infty)}(r)
$$

with

$$
\begin{aligned}
A_{+1} & :=\frac{\Gamma(b-a+1) \Gamma(c-a-b)}{\Gamma(1-a) \Gamma(c-a)}, \\
B_{+1} & :=\frac{\Gamma(b-a+1) \Gamma(a+b-c)}{\Gamma(b) \Gamma(b-c+1)} .
\end{aligned}
$$

\section{Square-integrability conditions}

The asymptotic behavior of the solutions at the endpoint $s=1, r=\infty$ is consistent with (3.8a), and exactly as stated in the $\kappa=-1$ case in Eq. (5.7a). In brief, for $\nu \in(0,1) \cup$ $(1,2)$ both solutions are square integrable and $r=\infty$ is a limit circle. For $\nu=1$ or $\nu \geq 2$, only $R_{1(\infty)}(r)$ is square integrable and radial infinity is limit point.
At $s=\infty, r=0$, we have

$$
\begin{aligned}
& \left|R_{1(0)}(r)\right|^{2} \sim r^{-2-4 \operatorname{Re}(\Upsilon)}, \\
& \left|R_{2(0)}(r)\right|^{2} \sim r^{-2+4 \operatorname{Re}(\Upsilon)},
\end{aligned}
$$

and since $q(r) \sim r, r=0$ is limit point. When $\operatorname{Re}(\Upsilon)<0$, only $R_{1(0)}(r)$ is square integrable, while if $\operatorname{Re}(\Upsilon)>0$, then only $R_{2(0)}(r)$ is square integrable. However, directly from the definition of $\Upsilon$, given by (6.3e), it follows that $\operatorname{Re}(\Upsilon)$ is always non-negative if we choose the principal root.

We denote the most general square-integrable radial solutions that are suitable at singular endpoint $r=0$ and $r=\infty$, respectively, by $R_{0}(r)$ and $R_{\gamma}(r)$, defined by

$$
\begin{aligned}
& R_{0}(r):=R_{2(0)}(r), \quad \text { if } \operatorname{Re}(\Upsilon)>0 \\
& R_{\gamma}(r):=\cos (\gamma) R_{1(\infty)}(r)+\sin (\gamma) R_{2(\infty)}(r)
\end{aligned}
$$

The solution $R_{\gamma}$ satisfies the generalized Robin boundary conditions at radial infinity parametrized by $\gamma \in[0, \pi)$, as for the cases $\kappa=0$ and $\kappa=-1$.

\section{Symmetries under conjugation and reflection}

The parameters of the hypergeometric equation satisfy the same properties of the case $\kappa=-1$, see Eq. (5.9). As a consequence, the radial solutions satisfy

$R_{j\left(r_{0}\right)}(r, \bar{\omega})=\overline{R_{j\left(r_{0}\right)}(r, \omega)}$, for $j \in\{1,2\}$, and $r_{0} \in\{0, \infty\}$.

Accordingly, we have for the general square-integrable solutions (6.9)

$$
\begin{aligned}
& R_{0}(r, \bar{\omega})=\overline{R_{0}(r, \omega)}, \\
& R_{\gamma}(r, \bar{\omega})=\overline{R_{\gamma}(r, \omega)} .
\end{aligned}
$$

\section{E. The radial Green function}

Considering the range of values $\nu \in(0,1) \cup(1,2)$ and $p(r)$ as in $(3.10 \mathrm{~d})$, the radial Green function $\mathcal{G}_{\omega}\left(r, r^{\prime}\right)$ for $\kappa=+1$ is written in terms of the radial solutions given by Eq. (6.9a) and (6.9b), as 


$$
\begin{aligned}
\mathcal{G}_{\omega}\left(r, r^{\prime}\right)= & \frac{1}{\mathcal{N}_{\omega}}\left(\Theta\left(r^{\prime}-r\right) R_{0}(r) R_{\gamma}\left(r^{\prime}\right)\right. \\
& \left.+\Theta\left(r-r^{\prime}\right) R_{0}\left(r^{\prime}\right) R_{\gamma}(r)\right),
\end{aligned}
$$

with normalization

$$
\mathcal{N}_{\omega}:=-p(r) W_{r}\left[R_{0}(r), R_{\gamma}(r)\right] .
$$

The Green function and its normalization are defined for all frequencies such that $\operatorname{Re}(\Upsilon)>0$. If we always choose the principle root of a complex number, then, $\operatorname{Re}(\Upsilon)$ is never negative, and it holds that:

$$
\begin{aligned}
& \operatorname{Re}(\Upsilon)=0 \\
& \Leftrightarrow \begin{cases}\omega=\Re(\omega) \in\left(\infty,-\omega_{c}\right] \cup\left[+\omega_{c}, \infty\right) & \text { and } \lambda<\frac{1}{2}, \\
\omega=\Re(\omega) & \text { and } \lambda=\frac{1}{2}, \\
\omega=\Re(\omega) \neq 0 & \text { and } \lambda>\frac{1}{2}, \\
\omega=i \operatorname{Im}(\omega) \in\left[-\omega_{c},+\omega_{c}\right] & \text { and } \lambda>\frac{1}{2},\end{cases}
\end{aligned}
$$

where the parameter $\omega_{c}$ is defined as:

$$
\omega_{c}=\frac{\sqrt{|1-2 \lambda|}}{2 L} .
$$

Since $\lambda=\lambda_{\ell}^{m}+\xi \in(-\infty, \xi)$, the conditions in Eq. (6.15) imply that $\xi \geq \frac{1}{2}$ gives rise to a $\lambda$-mode dependent domain for $\mathcal{N}_{\omega}$, which also entails $\lambda$-mode dependent boundary conditions. In this case, the existence of bound states depends crucially on the values of $\mu_{0}$ and $\xi$, as it did on the hyperbolic Lifshitz black hole. A preliminary analysis showed that for $\xi>1$, bound states emerge even for Dirichlet boundary conditions. For simplicity, in the following, we expose a detailed study considering $\xi<\frac{1}{2}$, which restricts the mass of the field in agreement with Eq. (3.9). Accordingly, $D\left(\mathcal{N}_{\omega}\right)$, the domain of $\mathcal{N}_{\omega}$ reads

$D\left(\mathcal{N}_{\omega}\right)=\mathbb{C} \backslash\left\{\omega|| \operatorname{Re}(\omega) \mid \geq \omega_{c}\right.$ and $\left.\operatorname{Im}(\omega)=0\right\}$.

In order to give an explicit expression of $\mathcal{N}_{\omega}$, observe that a square-integrable solution for $\operatorname{Re}(\Upsilon)>0$ can be written as a linear combination of the solutions at radial infinity as per Eq. (6.5). Using Eq. (6.5) and the Wronskian [29] (15.10.5), the normalization (6.14) reads

$$
\mathcal{N}_{\omega}=L^{4} \nu\left\{B_{+1} \cos (\gamma)-A_{+1} \sin (\gamma)\right\} .
$$

Since the coefficients above are such that $A_{+1}(\bar{\omega})=$ $\overline{A_{+1}(\omega)}$ and $B_{+1}(\bar{\omega})=\overline{B_{+1}(\omega)}$, we also have

$$
\mathcal{N}_{\bar{\omega}}=\overline{\mathcal{N}_{\omega}} \quad \text { and } \quad \mathcal{G}_{\bar{\omega}}=\overline{\mathcal{G}_{\omega}} .
$$

\section{F. On the existence of bound states}

In this section, we study the existence of bound states for $\xi<\frac{1}{2}$ and $\operatorname{Re}(\Upsilon)>0$, which yields (6.17). We consider the cases of Dirichlet, Neumann, and Robin boundary conditions separately, taking $n \in \mathbb{N}_{0}$.

\section{Dirichlet boundary condition, $\gamma=0$}

$$
\begin{aligned}
\mathcal{N}_{\omega}=0 & \Leftrightarrow\{b=-n \quad \text { or } \quad b-c+1\} \\
\Leftrightarrow & \omega= \pm \frac{i\left(2 \lambda+\nu^{2}+2 \nu+4 n^{2}+4 \nu n+4 n\right)}{4 L(\nu+2 n+1)} \\
& \text { and } \lambda \geq \lambda_{n},
\end{aligned}
$$

where

$$
\lambda_{n}:=\frac{2+4 n+4 n^{2}+2 \nu+4 n \nu+\nu^{2}}{2} .
$$

Since $\lambda=\lambda_{\ell}^{m}+\xi \leq \xi<\frac{1}{2}$, and clearly $\lambda_{n}>1$, the frequencies above do not represent poles of the radial Green function. Yet, it is easy to see that if $\xi>1$, then poles would emerge even for Dirichlet boundary condition, depending on the value of the other parameters.

\section{Neumann boundary condition, $\gamma=\pi / 2$}

$$
\mathcal{N}_{\omega}=0 \Leftrightarrow(1-a=-n \text { or } c-a=-n) .
$$

Defining the auxiliary quantities

$$
\begin{gathered}
\omega_{n}:=\frac{i\left(2 \lambda+\nu^{2}-2 \nu+4 n^{2}-4 \nu n+4 n\right)}{4 L(-\nu+2 n+1)}, \\
\lambda_{n}:=\frac{2+4 n+4 n^{2}-2 \nu-4 n \nu+\nu^{2}}{2},
\end{gathered}
$$

we find that

$1-a=0 \Leftrightarrow \omega=-\omega_{n=0}$ and $\begin{cases}\lambda \geq \lambda_{n=0}, & \text { for } \nu \in(0,1), \\ \lambda \leq \lambda_{n=0}, & \text { for } \nu \in(1,2) .\end{cases}$

$c-a=0 \Leftrightarrow \omega=+\omega_{n=0}$ and $\begin{cases}\lambda \geq \lambda_{n=0}, & \text { for } \nu \in(0,1), \\ \lambda \leq \lambda_{n=0}, & \text { for } \nu \in(1,2) .\end{cases}$

$$
\begin{aligned}
& 1-a=-n \neq 0 \Leftrightarrow \omega=-\omega_{n} \quad \text { and } \quad \lambda \geq \lambda_{n}, \\
& c-a=-n \neq 0 \Leftrightarrow \omega=+\omega_{n} \quad \text { and } \quad \lambda \geq \lambda_{n} .
\end{aligned}
$$

For $\nu \in(0,1) \cup(1,2)$, it holds that $\lambda_{n=0}>\frac{1}{2}$, as illustrated in Fig. 6. Moreover, $\lambda_{n}$ is an increasing function of $n$ since 


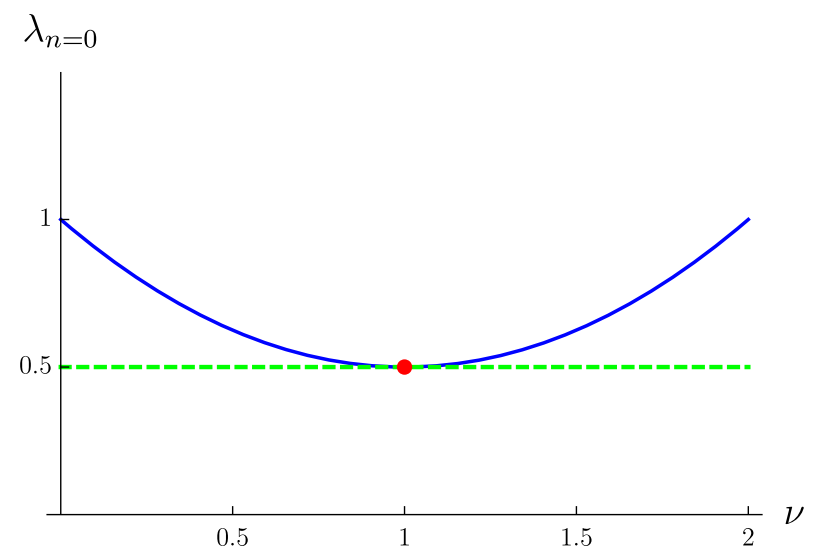

FIG. 6. $\lambda_{n=0}$ as a function of $\nu$. The (red) dot has coordinates $(1,0.5)$.

$\left.\frac{d \lambda_{n}}{d n}\right|_{n>0}=\left.(2-2 \nu+4 n)\right|_{n>0}>0$. We conclude that for $n=0$, the normalization has no zeros for $\nu \in(0,1)$, and has two zeros at $\omega= \pm \omega_{n=0}$ for $\nu \in(1,2)$. On the other hand, for $n>0$, there are no zeros for any value of $\nu$.

\section{Robin boundary conditions, $\gamma \in(\mathbf{0}, \pi / 2) \cup(\pi / 2, \pi)$}

Analogously to the case $\kappa=-1$, and using the same notation, define

$$
\begin{gathered}
\zeta(\omega):=\tan (\gamma)-\eta(\omega), \\
\eta(\omega):=\frac{\Gamma(a+b-c)}{\Gamma(c-a-b)} \frac{\Gamma(1-a) \Gamma(c-a)}{\Gamma(b) \Gamma(b-c+1)} .
\end{gathered}
$$

Observe that $\eta(\omega)$ is real valued whenever $\omega^{2} \in \mathbb{R}$, as illustrated in Fig. 7. In addition,

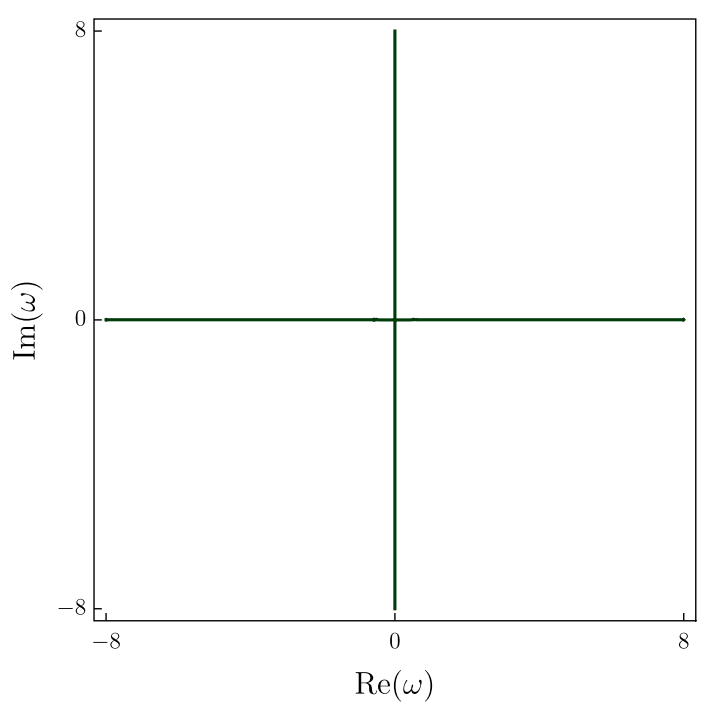

FIG. 7. Contour lines of $\operatorname{Im}(\eta)=0$ for $\mu_{0}=0, L=1, \lambda_{\ell}^{m}=0$, and $\xi=\frac{7}{88}$.

$$
\begin{aligned}
& \lim _{\omega \rightarrow 0} \eta(\omega)=\frac{\Gamma(\nu)}{\Gamma(-\nu)} \frac{\Gamma\left(\frac{1-\nu}{2}+\frac{\sqrt{1-2 \lambda}}{2}\right)^{2}}{\Gamma\left(\frac{1+\nu}{2}+\frac{\sqrt{1-2 \lambda}}{2}\right)^{2}} \\
& \lim _{\omega \rightarrow \omega_{p}} \eta(\omega)=\infty \\
& \lim _{\omega \rightarrow \infty} \eta(\omega)=0 \\
& \lim _{\omega \rightarrow \omega_{c}} \eta(\omega)=\frac{\Gamma(\nu)}{\Gamma(-\nu)} \frac{\Gamma\left(\frac{1-\nu}{2}-i \frac{\sqrt{1-2 \lambda}}{2}\right) \Gamma\left(\frac{1-\nu}{2}+i \frac{\sqrt{1-2 \lambda}}{2}\right)}{\Gamma\left(\frac{1+\nu}{2}-i \frac{\sqrt{1-2 \lambda}}{2}\right) \Gamma\left(\frac{1+\nu}{2}+i \frac{\sqrt{1-2 \lambda}}{2}\right)} .
\end{aligned}
$$

Note that the first limit, $\eta(0)$, is negative for $\nu \in(0,1)$ and positive for $\nu \in(1,2)$. The level sets of $\eta$ for $\kappa=+1$ are similar to the ones obtained in the previous two cases. However, in this case, the normalization has zeros not only for $\omega=i \operatorname{Im}(\omega)$ with $\operatorname{Im}(\omega)<0$, but also with $\operatorname{Im}(\omega)>0$, and for real frequencies as well. For $\operatorname{Im}(\omega)<0, \eta(i \operatorname{Im}(\omega))$ behaves like the function $\Xi(i \operatorname{Im}(\omega))$, as in Fig. 3 for $\nu \in(0,1)$, and as illustrated in Fig. 4 for $\nu \in(1,2)$. For $\operatorname{Im}(\omega)>0$, we have: $\eta(i \operatorname{Im}(\omega))=\eta(-i \operatorname{Im}(\omega))$. The zeros associated to the real frequencies, namely $\omega=\operatorname{Re}(\omega) \in$ $\left(-\omega_{c}, \omega_{c}\right)$ for $\omega_{c}$ as in (6.16), yield an additional interval of boundary conditions for which there are bound states, given by

$$
\gamma \in\left(\arctan \left(\eta\left(\omega_{c}\right)+\pi\right), \arctan (\eta(0)+\pi)\right) .
$$

Figures 8 and 9 illustrate the behavior of $\eta(\omega)$, for $\nu \in$ $(0,1)$ and $\nu \in(1,2)$, respectively, and including both curves for $\omega=i \operatorname{Im}(\omega)$ and $\omega=\operatorname{Re}(\omega)$.

In conclusion, for $\nu \in(1,2)$, we can impose either Dirichlet boundary conditions on all modes, or we can choose mode-dependent boundary conditions that respect the regime for which there are no bound states

$\gamma \in\left[0, \gamma_{c}^{\lambda}\right), \quad$ with $\quad \gamma_{c}^{\lambda}:=\arctan \left(\eta\left(\omega_{c}\right)\right) \in(0, \pi)$.

For $\nu \in(0,1)$, there are no bound states for $\gamma \in[0, \pi / 2]$ and we can also impose the mode-dependent boundary conditions as given above by Eq. (6.32).

\section{TWO-POINT FUNCTIONS FOR GENERAL BOUNDARY CONDITIONS}

In this section, we address the question whether one can construct two-point functions of a free, scalar, massive quantum field theory on Lifshitz spacetime and on Lifshitz topological black holes for the admissible class of boundary condition of Robin type individuated in the previous sections. Inasmuch as they are static spacetimes, one can follow the same construction used [10-15,28]. More specifically, the underlying global, timelike Killing field 


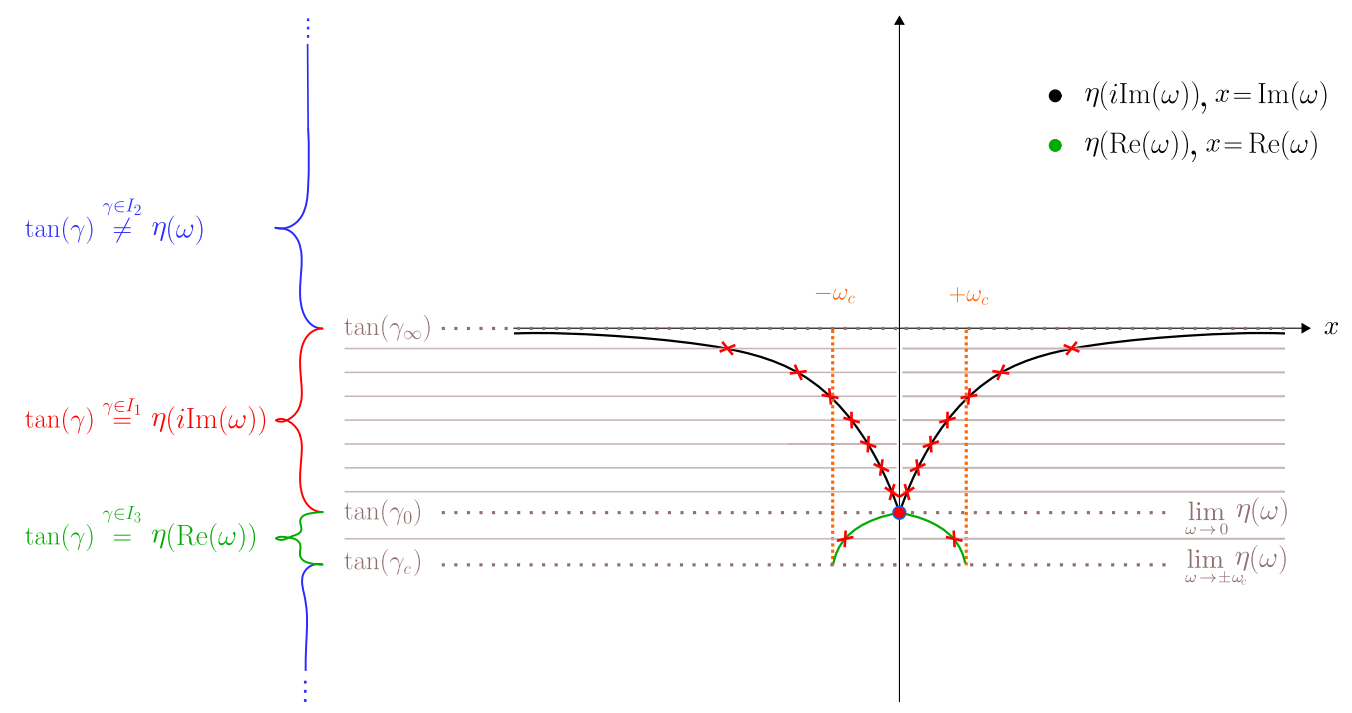

FIG. 8. The behavior of $\eta$ for frequencies such that $\omega^{2} \in \mathbb{R}$ and $\nu \in(0,1)$.

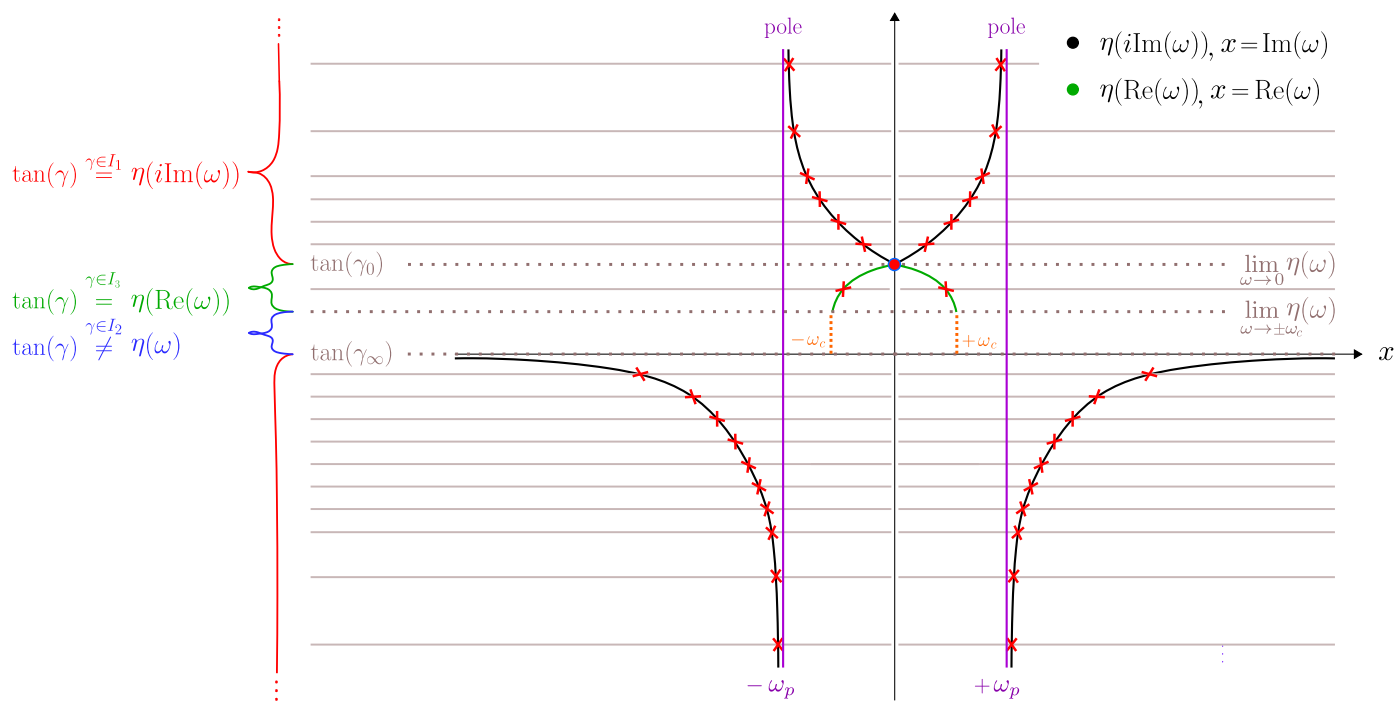

FIG. 9. The behavior of $\eta$ for frequencies such that $\omega^{2} \in \mathbb{R}$ and $\nu \in(1,2)$. Note that $\left|\omega_{c}\right|$ might be larger than $\left|\omega_{p}\right|$, i.e., in this illustration, the $x$-axis does not necessarily have the same scale for $x=\Re(\omega)$ and $x=\operatorname{Im}(\omega)$.

identifies a Hamiltonian and, accordingly, one can select an associated ground state for each admissible boundary condition. Using general properties of quantum field on curved backgrounds, one can infer a priori that such states are physically sensible, namely they satisfy the local Hadamard condition, due to a general result of Sahlmann and Verch [32]. Here we do not enter into the details of such property, leaving an interested reader to the review [33] or to [34].

First, in Sec. VII A, we describe the framework of the construction of the two-point functions, which apply to all spacetimes considered in this work. Then, we employ it explicitly on Lifshitz spacetime, on a hyperbolic and on a spherical Lifshitz topological black hole, respectively, in Secs. VII B, VII C, and VII D.

\section{A. The construction of physically sensible two-point functions}

On Lif ${ }_{K}$, a two-point function of a free, scalar, massive Klein-Gordon theory is a bidistribution $G^{+} \in \mathcal{D}^{\prime}\left(\operatorname{Lif}_{K} \times\right.$ Lif $_{\kappa}$ ) that is positive

$$
G^{+}(f, f) \geq 0, \quad \forall f \in C_{0}^{\infty}\left(\operatorname{Lif}_{\kappa}\right)
$$

and solves the Klein-Gordon equation in each entry

$$
(P \otimes \mathbb{1}) G^{+}\left(f, f^{\prime}\right)=(\mathbb{1} \otimes P) G^{+}\left(f, f^{\prime}\right)=0,
$$

for $P$ as in Eq. (3.3) and $f, f^{\prime} \in C_{0}^{\infty}\left(\mathrm{Lif}_{\kappa}\right)$. If $E$ is the causal propagator, defined as the Green function of the 
Klein-Gordon equation, then the antisymmetric part of $G^{+}$satisfies

$$
i E\left(f, f^{\prime}\right)=G^{+}\left(f, f^{\prime}\right)-G^{+}\left(f^{\prime}, f\right) .
$$

To guarantee that the canonical commutation relations of the underlying quantum field theory hold, we impose the initial conditions-see, for example [34], Ch. 3-at the level of integral kernel

$$
\begin{gathered}
\left.E\left(x, x^{\prime}\right)\right|_{t=t^{\prime}}=0 \\
\left.\partial_{t} E\left(x, x^{\prime}\right)\right|_{t=t^{\prime}}=-\left.\partial_{t^{\prime}} E\left(x, x^{\prime}\right)\right|_{t=t^{\prime}}=\frac{\delta\left(\underline{x}, \underline{x}^{\prime}\right)}{q(x)},
\end{gathered}
$$

where $x=(t, \underline{x}) \in \mathrm{Lif}_{\kappa}$ and where $q$ is the function introduced in Eq. (3.10c). An analogous expression holds true for $x^{\prime}$. Given that $\mathcal{M}$ admits a Killing field in the time direction, analogously to the ansatz for $\Psi$ (3.4), we consider the ansatz for the integral kernel of $G^{+}$

$$
\begin{aligned}
G^{+}\left(x, x^{\prime}\right)= & \lim _{\varepsilon \rightarrow 0^{+}} \int_{\sigma(\Delta)} d \Sigma(\ell, m) \\
& \times \int_{0}^{\infty} d \omega e^{-i \omega\left(t-t^{\prime}-i \varepsilon\right)} \hat{G}_{\omega}\left(r, r^{\prime}\right) \\
& \times Y_{\ell}^{m}(\theta, \varphi) Y_{\ell}^{m}\left(\theta^{\prime}, \varphi^{\prime}\right)
\end{aligned}
$$

where $i \varepsilon$ is a standard regularization, the limit is understood in the weak sense, $Y_{\ell}^{m}(\theta, \varphi)$ are the real-valued eigenfunctions of the Laplacian operator $\Delta$ with spectrum $\sigma(\Delta)$ and associated measure $d \Sigma(\ell, m)$, as mentioned in Sec. III. For convenience, we take the harmonics normalized such that their completeness relation reads:

$$
\int_{\sigma(\Delta)} d \Sigma(\ell, m) Y_{\ell}^{m}(\theta, \varphi) Y_{\ell}^{m}\left(\theta^{\prime}, \varphi^{\prime}\right)=\delta\left(\theta-\theta^{\prime}\right) \delta\left(\varphi-\varphi^{\prime}\right) .
$$

With the ansatz (7.5), the first initial condition, given by Eq. (7.4a), is automatically satisfied if $\hat{G}_{\omega}\left(r, r^{\prime}\right)=$ $\hat{G}_{\omega}\left(r^{\prime}, r\right)$. Furthermore, if $\hat{G}_{\omega}\left(r, r^{\prime}\right)$ is also invariant under the mapping $\omega \mapsto-\omega$, and taking into account the completeness relations of the harmonics, then condition (7.4b) yields

$$
\int_{\mathbb{R}} d \omega \omega \hat{G}_{\omega}\left(r, r^{\prime}\right)=\frac{\delta\left(r-r^{\prime}\right)}{q(r)} .
$$

At the same time, for $L_{\omega^{2}}$ as the Sturm-Liouville operator defined in $(3.10 \mathrm{~b})$, the radial Green function $\mathcal{G}_{\omega}$ is a solution of

$$
\begin{aligned}
\left(\left(L_{\omega^{2}}-\omega^{2}\right) \otimes \mathbb{1}\right) \mathcal{G}_{\omega}\left(r, r^{\prime}\right) & =\left(\mathbb{1} \otimes\left(L_{\omega^{2}}-\omega^{2}\right)\right) \mathcal{G}_{\omega}\left(r, r^{\prime}\right) \\
& =\frac{\delta\left(r-r^{\prime}\right)}{q(r)},
\end{aligned}
$$

with measure $q(r)$ defined in (3.10c). In addition, the spectral resolution of the radial Green function [27,31] reads

$$
\begin{aligned}
& \frac{1}{2 \pi i} \oint_{\mathcal{C}^{\infty}} d\left(\omega^{2}\right) \mathcal{G}_{\omega}\left(r, r^{\prime}\right)+\frac{1}{2 \pi i} \sum_{\text {poles }} \operatorname{Res}\left[\mathcal{G}_{\omega}\left(r, r^{\prime}\right)\right] \\
& \quad=-\frac{\delta\left(r-r^{\prime}\right)}{s(r)}
\end{aligned}
$$

where $\mathcal{C}^{\infty}$ corresponds to a contour that, in the limit of infinite radius, covers the entire region on the $\omega^{2}$-complex plane where $\mathcal{G}_{\omega}$ is meromorphic. Note that when the radial Green function has no poles, the summation vanishes, and, if we can reduce the contour integral to an integral over the real line, we can obtain $\hat{G}_{\omega}\left(r, r^{\prime}\right)$ directly by comparing (7.7) with (7.9)

$$
\int_{\mathbb{R}} d \omega \omega \hat{G}_{\omega}\left(r, r^{\prime}\right)=-\frac{1}{2 \pi i} \oint_{\mathcal{C}^{\infty}} d\left(\omega^{2}\right) \mathcal{G}_{\omega}\left(r, r^{\prime}\right) .
$$

Subsequently, for each spacetime $\mathrm{Lif}_{\kappa}$, we write the spectral resolution of the radial Green function, identify the appropriate contour, and show that it can be reduced to an integral over real frequencies. With that, we extract a suitable definition of the only remaining unknown function $\hat{G}_{\omega}\left(r, r^{\prime}\right)$. A two-point function as constructed above, of the form (7.5), satisfies the canonical commutation relations and characterizes a ground-state of local Hadamard form [32]. With such ground state in hands, a thermal state can be directly constructed by replacing the time-dependent function. That is, if the two-point function of the ground state (7.5) is well defined, then a KMS state at inverse temperature $\beta$ with respect to $\partial_{t}$ is characterized by twopoint function of the form

$$
\begin{aligned}
G_{\beta}^{+}\left(x, x^{\prime}\right)= & \lim _{\varepsilon \rightarrow 0^{+}} \int_{\sigma(\Delta)} d \Sigma(\ell, m) \int_{0}^{\infty} d \omega T_{\omega}\left(t, t^{\prime}\right) \\
& \times \hat{G}_{\omega}\left(r, r^{\prime}\right) Y_{\ell}^{m}(\theta, \varphi) Y_{\ell}^{m}\left(\theta^{\prime}, \varphi^{\prime}\right),
\end{aligned}
$$

with

$$
T_{\omega}\left(t, t^{\prime}\right):=\frac{e^{\beta \omega} e^{-i \omega\left(t-t^{\prime}-i \varepsilon\right)}+e^{+i \omega\left(t-t^{\prime}+i \varepsilon\right)}}{e^{\beta \omega}-1} .
$$

\section{B. Two-point functions on Lifshitz spacetime Lif $_{\mathbf{0}}$}

Bearing in mind the analysis of Sec. IV, for an effective mass such that $\nu \in(0,1) \cup(1,2)$, and for boundary conditions parametrized by $\gamma$ at radial infinity, the radial Green function on $\mathrm{Lif}_{0}$, given by Eq. (4.22), reads 


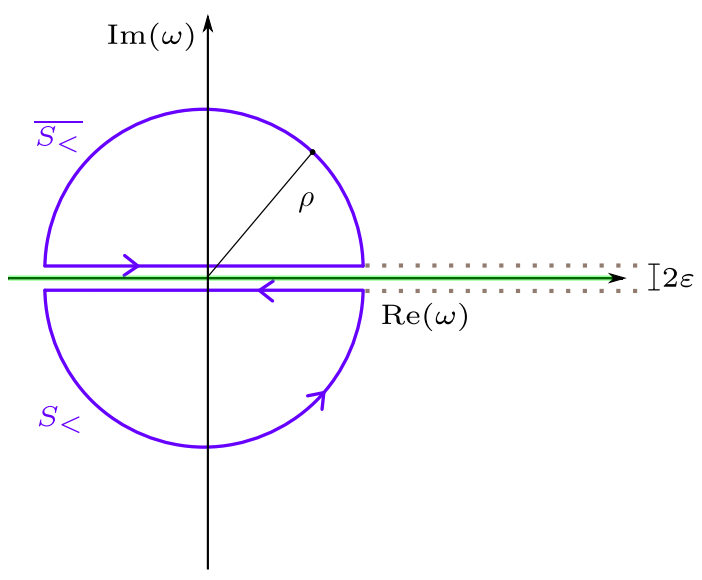

FIG. 10. Contour of two semicircles on the $\omega$-complex plane.

$$
\begin{aligned}
\mathcal{G}_{\omega}\left(r, r^{\prime}\right)= & \frac{1}{\mathcal{N}_{\omega}}\left(\Theta\left(r^{\prime}-r\right) R_{0}(r) R_{\gamma}\left(r^{\prime}\right)\right. \\
& \left.+\Theta\left(r-r^{\prime}\right) R_{0}\left(r^{\prime}\right) R_{\gamma}(r)\right) .
\end{aligned}
$$

Recalling that $R_{0}(r)$ is defined by parts, we define $\mathcal{G}_{\omega}^{<}\left(r, r^{\prime}\right)$ and $\mathcal{G}_{\omega}^{>}\left(r, r^{\prime}\right)$ implicitly by

$$
\begin{aligned}
\mathcal{G}_{\omega}\left(r, r^{\prime}\right)= & \Theta(-\operatorname{Im}(\omega)) \mathcal{G}_{\omega}^{<}\left(r, r^{\prime}\right) \\
& +\Theta(\operatorname{Im}(\omega)) \mathcal{G}_{\omega}^{>}\left(r, r^{\prime}\right) .
\end{aligned}
$$

In view of the symmetry properties described in Sec. IV D, it holds

$$
\overline{\mathcal{G}_{\omega}^{<}\left(r, r^{\prime}\right)}=\mathcal{G}_{\bar{\omega}}^{>}\left(r, r^{\prime}\right)
$$

For $\nu \in(0,1)$ and $\gamma \in[0, \pi / 2]$, the radial Green function has no poles, but it is not defined for frequencies with $\operatorname{Im}(\omega)=0$. Therefore, on the $\omega$-complex plane, the suitable contour of the spectral resolution (7.9) consists of two semicircles, as in Fig. 10. Taking into account Eq. (7.13), we write

$$
\begin{aligned}
& \oint_{\mathcal{C}^{\infty}} d \omega \omega \mathcal{G}_{\omega}\left(r, r^{\prime}\right) \\
& \quad=\lim _{\varepsilon \rightarrow 0} \lim _{\rho \rightarrow \infty}\left\{\oint_{S_{<}} d \omega \omega \mathcal{G}_{\omega}^{<}\left(r, r^{\prime}\right)+\oint_{\overline{S_{<}}} d \omega \omega \overline{\mathcal{G}_{\bar{\omega}}^{<}\left(r, r^{\prime}\right)}\right\} .
\end{aligned}
$$

Each contour integral of the expression above has two components given by the right and left propagating terms of $\mathcal{G}_{\omega}$. For $\operatorname{Im}(\omega)<0$, we define the right component as

$$
\mathcal{G}_{\omega}^{<, R}\left(r, r^{\prime}\right)=\frac{1}{\mathcal{N}_{\omega}} \Theta\left(r^{\prime}-r\right) R_{1(0)}(r) R_{\gamma}\left(r^{\prime}\right),
$$

while $\mathcal{G}_{\omega}^{<, L}\left(r, r^{\prime}\right)=\mathcal{G}_{\omega}^{<}\left(r, r^{\prime}\right)-\mathcal{G}_{\omega}^{<, R}\left(r, r^{\prime}\right)$. Therefore, the right-hand side of Eq. (7.29) can be decomposed as

$$
\begin{gathered}
\lim _{\varepsilon \rightarrow 0} \lim _{\rho \rightarrow \infty}\left\{\oint_{S_{<}} d \omega \omega\left[\mathcal{G}_{\omega}^{<, R}\left(r, r^{\prime}\right)+\mathcal{G}_{\omega}^{<, L}\left(r, r^{\prime}\right)\right]\right. \\
+\oint_{\overline{S_{<}}} d \omega \omega\left[\overline{\mathcal{G}_{\bar{\omega}}^{<, R}\left(r, r^{\prime}\right)}+\overline{\left.\mathcal{G}_{\bar{\omega}}^{<, L}\left(r, r^{\prime}\right)\right]}\right\} .
\end{gathered}
$$

First, let us focus on the integration over the right components. That is, consider

$I^{R}:=\oint_{S_{<}} d \omega \omega \mathcal{G}_{\omega}^{<, R}\left(r, r^{\prime}\right)+\oint_{\overline{S_{<}}} d \omega \omega \overline{\mathcal{G}_{\bar{\omega}}^{<, R}\left(r, r^{\prime}\right)}$

Since the integrand has no poles within the contour, by the same argument of [13], Appendix A, or by a direct application of Jordan's Lemma, the arc integrals vanish. Thus, substituting the definitions and recalling that the radial solutions satisfy properties (4.16), it follows:

$$
\lim _{\varepsilon \rightarrow 0} \lim _{\rho \rightarrow \infty} I^{R}=\left.\Theta\left(r^{\prime}-r\right) \int_{\mathbb{R}} d \tilde{\rho} \tilde{\rho}\left[\left(\frac{1}{\mathcal{N}_{\omega}} R_{1(0)}(r)-\frac{1}{\overline{\mathcal{N}_{\omega}}} \overline{R_{1(0)}(r)}\right) R_{\gamma}\left(r^{\prime}\right)\right]\right|_{\omega=\tilde{\rho}} .
$$

Using the expressions (4.6a), (4.24), and (4.26), we simplify the term within the brackets in Eq. (7.18) and the contribution to the contour integral coming from the right propagating terms is

$$
\lim _{\varepsilon \rightarrow 0} \lim _{\rho \rightarrow \infty} I^{R}=\left.\Theta\left(r^{\prime}-r\right) \int_{\mathbb{R}} d \tilde{\rho} \tilde{\rho}\left[\frac{1}{4 \nu} \frac{A_{0} \overline{B_{0}}-B_{0} \overline{A_{0}}}{\left|B_{0} \cos (\gamma)-A_{0} \sin (\gamma)\right|^{2}} R_{\gamma}(r) R_{\gamma}\left(r^{\prime}\right)\right]\right|_{\omega=\tilde{\rho}} .
$$

We can obtain the contribution from the left propagating terms by an analogous computation. However, noting that the integrand above is invariant under the mapping $r \leftrightarrow r^{\prime}$, we can see that it is not necessary to compute it, and that adding both contributions is equivalent to scratching out the Heaviside function of the right-hand side of (7.19). 
All in all, we obtain

$$
\begin{aligned}
& \oint_{\mathcal{C}^{\infty}} d \omega \omega \mathcal{G}_{\omega}\left(r, r^{\prime}\right) \\
& \quad=\int_{\mathbb{R}} d \omega \omega\left[\frac{1}{4 \nu} \frac{A_{0} \overline{B_{0}}-B_{0} \overline{A_{0}}}{\left|B_{0} \cos (\gamma)-A_{0} \sin (\gamma)\right|^{2}} R_{\gamma}(r) R_{\gamma}\left(r^{\prime}\right)\right],
\end{aligned}
$$

which yields, by comparing it with Eq. (7.10), the function:

$$
\hat{G}_{\omega}\left(r, r^{\prime}\right)=\frac{1}{4 \pi \nu} \frac{\operatorname{Im}\left(B_{0} \overline{A_{0}}\right)}{\left|B_{0} \cos (\gamma)-A_{0} \sin (\gamma)\right|^{2}} R_{\gamma}(r) R_{\gamma}\left(r^{\prime}\right) .
$$

Note that $\hat{G}_{\omega}\left(r, r^{\prime}\right)$ is, as needed to guarantee the canonical commutation relations, invariant under both transformations $\omega \leftrightarrow-\omega$ and $r \leftrightarrow r^{\prime}$.

Let $Y_{\ell}^{m}(\theta, \varphi)$ be the real-valued eigenfunctions of the Laplacian operator on the two-dimensional Euclidean space. A two-point function with integral kernel

$$
\begin{aligned}
G^{+}\left(x, x^{\prime}\right)= & \lim _{\varepsilon \rightarrow 0^{+}} \int_{\mathbb{R}} d \ell \int_{\mathbb{R}} d m \int_{0}^{\infty} d \omega e^{-i \omega\left(t-t^{\prime}-i \varepsilon\right)} \\
& \times \hat{G}_{\omega}\left(r, r^{\prime}\right) Y_{\ell}^{m}(\theta, \varphi) Y_{\ell}^{m}\left(\theta^{\prime}, \varphi^{\prime}\right)
\end{aligned}
$$

characterizes a ground state for the free, scalar, massive Klein-Gordon field on a Lifshitz spacetime. By the same token, a two-point of the form

$$
\begin{aligned}
G_{\beta}^{+}\left(x, x^{\prime}\right)= & \lim _{\varepsilon \rightarrow 0^{+}} \int_{\mathbb{R}} d \ell \int_{\mathbb{R}} d m \int_{0}^{\infty} d \omega T_{\omega}\left(t, t^{\prime}\right) \\
& \times \hat{G}_{\omega}\left(r, r^{\prime}\right) Y_{\ell}^{m}(\theta, \varphi) Y_{\ell}^{m}\left(\theta^{\prime}, \varphi^{\prime}\right),
\end{aligned}
$$

with $T_{\omega}\left(t, t^{\prime}\right)$ given by (7.11b), characterizes a KMS state at inverse-temperature $\beta$ with respect to the Killing field $\partial_{t}$ for the free, scalar, massive Klein-Gordon field on a Lifshitz spacetime. Both two-point functions are of local Hadamard form and satisfy the canonical commutation relations.

\section{Two-point functions on a hyperbolic Lifshitz black hole Lif $_{-1}$}

On the hyperbolic Lifshitz black hole, Lif ${ }_{-1}$, the computation regarding the spectral decomposition of the radial Green function is equivalent to the one described in the previous section, for the $\kappa=0$ case. The radial Green function is also defined by parts with respect to the sign of the imaginary part of the frequencies, the suitable contour is also given by the one in Fig. 10 and the steps to follow are the same. Invoking the results of Sec. V regarding the Klein-Gordon equation, we consider values of mass for which no bound states emerge, as given in (5.24), and the acceptable boundary conditions, as described in Sec. V F. Analogously to (7.31), we obtain

$$
\hat{G}_{\omega}\left(r, r^{\prime}\right)=\frac{1}{\pi L^{4} \nu} \frac{\operatorname{Im}\left(B_{-1} \overline{A_{-1}}\right)}{\left|B_{-1} \cos (\gamma)-A_{-1} \sin (\gamma)\right|^{2}} R_{\gamma}(r) R_{\gamma}\left(r^{\prime}\right) .
$$

With $\hat{G}_{\omega}\left(r, r^{\prime}\right)$ as above, $T_{\omega}\left(t, t^{\prime}\right)$ as in $(7.11 \mathrm{~b})$ and letting $Y_{\ell}^{m}(\theta, \varphi)$ be the real-valued eigenfunctions of the Laplacian operator on the 2-dimensional hyperbolic space, it follows that:

$$
\begin{aligned}
G^{+}\left(x, x^{\prime}\right)= & \lim _{\varepsilon \rightarrow 0^{+}} \int_{0}^{\infty} d \ell \sum_{m=0}^{\infty} \int_{0}^{\infty} d \omega e^{-i \omega\left(t-t^{\prime}-i \varepsilon\right)} \\
& \times \hat{G}_{\omega}\left(r, r^{\prime}\right) Y_{\ell}^{m}(\theta, \varphi) Y_{\ell}^{m}\left(\theta^{\prime}, \varphi^{\prime}\right)
\end{aligned}
$$

and

$$
\begin{aligned}
G_{\beta}^{+}\left(x, x^{\prime}\right)= & \lim _{\varepsilon \rightarrow 0^{+}} \int_{0}^{\infty} d \ell \sum_{m=0}^{\infty} \int_{0}^{\infty} d \omega T_{\omega}\left(t, t^{\prime}\right) \\
& \times \hat{G}_{\omega}\left(r, r^{\prime}\right) Y_{\ell}^{m}(\theta, \varphi) Y_{\ell}^{m}\left(\theta^{\prime}, \varphi^{\prime}\right),
\end{aligned}
$$

are two-point functions that characterize, respectively, a ground state and a KMS state at inverse-temperature $\beta$ with respect to the Killing field $\partial_{t}$, for the free, scalar, massive Klein-Gordon field on a hyperbolic Lifshitz black hole, which are of local Hadamard form and satisfy the canonical commutation relations.

\section{Two-point functions on a spherical Lifshitz black hole Lif $_{1}$}

The spherical Lifshitz black hole constitutes a manifestly different scenario from the previous two cases. Recall that in this case the radial Green function is defined for all frequencies except for the real-valued ones with $|\omega|>\omega_{c}$, as stated in Eq. (6.15), which is tantamount to assume $\operatorname{Re} \Upsilon>0$ together with $\xi<\frac{1}{2}$. We consider the regime of boundary conditions for which there are no bound states, namely for $\nu \in(0,1)$ we take $\gamma \in[0, \pi / 2]$, and for

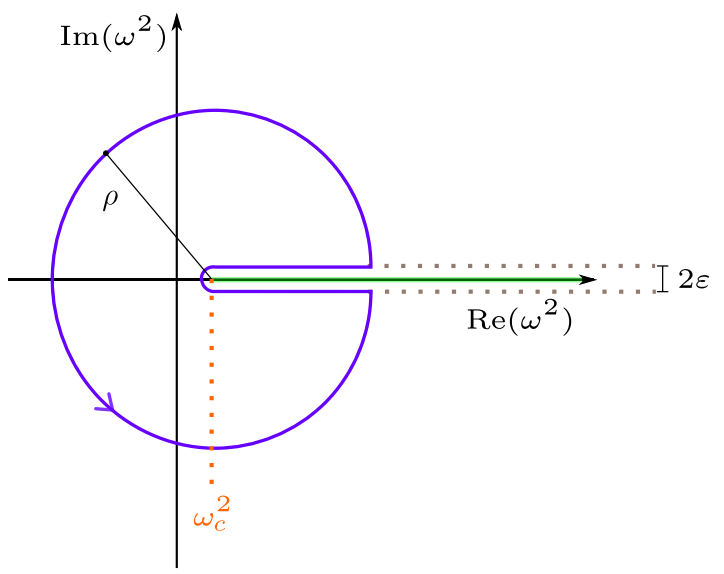

FIG. 11. "Pac-man" contour on the $\omega^{2}$-complex plane. 
$\nu \in(1,2)$, we assume either $\gamma=0$ or $\gamma$ is mode dependent, as detailed in Sec. VIF.

For $\operatorname{Re} \Upsilon>0$, the radial Green function, given by Eq. (6.13) with $R_{2(0)}(r)$ determined by (6.4), reads:

$$
\begin{aligned}
\mathcal{G}_{\omega}\left(r, r^{\prime}\right)= & \frac{1}{\mathcal{N}_{\omega}}\left(\Theta\left(r^{\prime}-r\right) R_{2(0)}(r) R_{\gamma}\left(r^{\prime}\right)\right. \\
& \left.+\Theta\left(r-r^{\prime}\right) R_{2(0)}\left(r^{\prime}\right) R_{\gamma}(r)\right) .
\end{aligned}
$$

Let us define the right propagating term by

$$
\mathcal{G}_{\omega}^{R}\left(r, r^{\prime}\right)=\frac{1}{\mathcal{N}_{\omega}} \Theta\left(r^{\prime}-r\right) R_{2(0)}(r) R_{\gamma}\left(r^{\prime}\right)
$$

and the left propagating term by $\mathcal{G}_{\omega}^{L}\left(r, r^{\prime}\right)=\mathcal{G}_{\omega}\left(r, r^{\prime}\right)-$ $\mathcal{G}_{\omega}^{R}\left(r, r^{\prime}\right)$. The suitable contour for the spectral resolution is given by the "pac-man" contour on the $\omega^{2}$-complex plane as illustrated in Fig. 11. As a consequence

$$
\begin{aligned}
& \oint_{\mathcal{C}^{\infty}} d\left(\omega^{2}\right) \mathcal{G}_{\omega}\left(r, r^{\prime}\right) \\
& =\lim _{\varepsilon \rightarrow 0} \lim _{\rho \rightarrow \infty} \oint_{\text {"pac-man" }} d\left(\omega^{2}\right)\left\{\mathcal{G}_{\omega}^{R}\left(r, r^{\prime}\right)+\mathcal{G}_{\omega}^{L}\left(r, r^{\prime}\right)\right\} .
\end{aligned}
$$

First, we note that the arc integrals vanish, as in the previous two cases. The integral over $\rho$ of the right propagating terms, denoted by $I^{R}$, yields

$$
\lim _{\varepsilon \rightarrow 0} \lim _{\rho \rightarrow \infty} I^{R}=\left.\Theta\left(r^{\prime}-r\right) \int_{+\omega_{c}^{2}}^{+\infty} d \tilde{\rho}\left\{\left(\frac{1}{\mathcal{N}_{\omega}} R_{2(0)}(r)-\frac{1}{\mathcal{N}_{\bar{\omega}}} \overline{R_{2(0)}(r)}\right) R_{\gamma}\left(r^{\prime}\right)\right\}\right|_{\omega=\sqrt{\tilde{\rho}}} .
$$

Defining $\tilde{\omega}^{2}=\omega^{2}-\omega_{c}^{2}$, which depends on $\lambda$ because $\omega_{c}$ does, and invoking the same arguments given in the previous two spacetimes, we obtain the function $\hat{G}_{\tilde{\omega}}\left(r, r^{\prime}\right)$ for the spherical case

$$
\hat{G}_{\tilde{\omega}}\left(r, r^{\prime}\right)=\left.\left[\frac{1}{\pi L^{4} \nu} \frac{\operatorname{Im}\left(B_{+1} \overline{A_{+1}}\right)}{\left|B_{+1} \cos (\gamma)-A_{+1} \sin (\gamma)\right|^{2}} R_{\gamma}(r) R_{\gamma}\left(r^{\prime}\right)\right]\right|_{\omega=\sqrt{\tilde{\omega}^{2}+\omega_{c}^{2}}} .
$$

Last, let $Y_{\ell}^{m}(\theta, \varphi)$ be the spherical harmonics and $T_{\tilde{\omega}}\left(t, t^{\prime}\right)$ as in (7.11b). Two-point functions with integral kernels given by

$$
G^{+}\left(x, x^{\prime}\right)=\lim _{\varepsilon \rightarrow 0^{+}} \sum_{\ell=0}^{\infty} \sum_{m=-\ell}^{\ell} \int_{0}^{\infty} d \tilde{\omega} e^{-i \tilde{\omega}\left(t-t^{\prime}-i \varepsilon\right)} \hat{G}_{\tilde{\omega}}\left(r, r^{\prime}\right) Y_{\ell}^{m}(\theta, \varphi) Y_{\ell}^{m}\left(\theta^{\prime}, \varphi^{\prime}\right),
$$

and

$$
G_{\beta}^{+}\left(x, x^{\prime}\right)=\lim _{\varepsilon \rightarrow 0^{+}} \sum_{\ell=0}^{\infty} \sum_{m=-\ell}^{\ell} \int_{0}^{\infty} d \tilde{\omega} T_{\tilde{\omega}}\left(t, t^{\prime}\right) \hat{G}_{\tilde{\omega}}\left(r, r^{\prime}\right) Y_{\ell}^{m}(\theta, \varphi) Y_{\ell}^{m}\left(\theta^{\prime}, \varphi^{\prime}\right),
$$

characterize, respectively, a ground and a KMS state at inverse-temperature $\beta$ with respect to the Killing field $\partial_{t}$ for the free, scalar, massive Klein-Gordon field on $\mathrm{Lif}_{1}$. Recall that, in addition, they are of local Hadamard form and they satisfy the canonical commutation relations per construction.

\section{CONCLUSION}

We showed that a free, scalar field with effective mass $\mu^{2} \in\left(-\frac{4}{L^{2}},-\frac{3}{L^{2}}\right)$ on a class of Lifshitz type spacetime, Lif $_{\kappa}$, can be endowed with a class of Robin boundary conditions ruled by a continuous parameter $\gamma \in[0, \pi / 2]$. Each of these boundary conditions yield nonequivalent dynamics and different two-point functions, thus generalizing the standard quantization procedure which relies on Dirichlet boundary conditions. From a physical viewpoint all these additional options appear to be a legitimate choice since, on the one hand, we are still describing a closed system, while, on the other hand, we can construct an associated two-point function, locally of Hadamard form. Hence, physical observables, such as the renormalized stress-energy tensor, can be constructed yielding finite expectation values and finite quantum fluctuations.

It is worth mentioning that one might foresee to consider a more general class of boundary conditions, much in the spirit of what happens on asymptotically AdS spacetimes, see e.g., [28,35]. In particular, for the range of effective mass $\mu^{2} \in\left(-\frac{4}{L^{2}},-\frac{3}{L^{2}}\right) \cup\left(-\frac{3}{L^{2}},-\frac{2}{L^{2}}\right), \lambda$-mode dependent boundary conditions are admissible. Similar options have been recently investigated in the context of AdS spacetimes [36]. Hence an analysis in this direction would be certainly 
an interesting research project, which we hope to undertake in the next future.

In addition we emphasize that we expect that the framework considered in this paper can be generalized to higher dimensional Lifshitz spacetimes of critical exponent $z=2$. For other values of $z>2$ a case-by-case study is necessary, since the classification, and hence the properties of the solutions of the radial part of the Klein-Gordon equation are strongly dependent on the choice of $z$. Moreover, starting from this work, one can conceive other three additional research avenues. First, one can perform a numerical analysis to understand how the choice of boundary conditions and of topology affects vacuum fluctuations, much in the same spirit of the work presented in [37] for topological black holes of Einstein gravity. Second, one can compute the transition rate of an Unruh-DeWitt detector in a $\mathrm{Lif}_{K}$ spacetime, following the same prescription of [15] unveiling in particular how such detector distinguishes among the three Lifshitz spacetimes considered, or how it interacts differently with a massless hyperbolic black hole or with a Lifshitz topological black holes. Third, one could consider asymptotically Lifshitz spacetimes with different interiors, such as $\mathrm{AdS}_{2} \times \mathbb{R}^{2}$, which are of particular relevance within the AdS/CFT framework [38,39]. Although all the structures and analyses discussed in the main body of this work can be in principle adapted to these cases, it is hard to use only analytic formulas, even in the study of the classical dynamics and one would be forced to rely heavily on numerical analyses. For this reason we decided to postpone the investigation of these scenarios to future works, focusing here only on specific and more symmetric backgrounds. This has the net advantage of allowing us to focus on highlighting the possibilities offered by considering a more general class of boundary conditions without being hindered by additional technical difficulties.

\section{ACKNOWLEDGMENTS}

The work of L. S.C is supported by a PhD scholarship of the University of Pavia, which is gratefully acknowledged. Part of this work is based on the MSc thesis of D.S. submitted for evaluation to the University of Pavia.
[1] P. Hořava, Phys. Rev. D 79, 084008 (2009).

[2] A. Wang, Int. J. Mod. Phys. D 26, 1730014 (2017).

[3] S. A. Hartnoll, Classical Quantum Gravity 26, 224002 (2009).

[4] P. Hořava, Classical Quantum Gravity 28, 114012 (2011).

[5] A. Giacomini, G. Giribet, M. Leston, J. Oliva, and S. Ray, Phys. Rev. D 85, 124001 (2012).

[6] S. Kachru, X. Liu, and M. Mulligan, Phys. Rev. D 78, 106005 (2008).

[7] G. M. Quinta, A. Flachi, and J. P. S. Lemos, Phys. Rev. D 93, 124073 (2016).

[8] A. Ishibashi and R. M. Wald, Classical Quantum Gravity 20, 3815 (2003).

[9] A. Ishibashi and R. M. Wald, Classical Quantum Gravity 21, 2981 (2004).

[10] C. Dappiaggi and H. Ferreira, Phys. Rev. D 94, 125016 (2016).

[11] C. Dappiaggi and H. Ferreira, Rev. Math. Phys. 30, 1850004 (2018).

[12] C. Dappiaggi, H. Ferreira, and A. Marta, Phys. Rev. D 98, 025005 (2018).

[13] F. Bussola, C. Dappiaggi, H. R. C. Ferreira, and I. Khavkine, Phys. Rev. D 96, 105016 (2017).

[14] F. Bussola and C. Dappiaggi, Classical Quantum Gravity 36, 015020 (2019).

[15] L. Campos and C. Dappiaggi, Phys. Rev. D 103, 025021 (2021).

[16] R. B. Mann, J. High Energy Phys. 06 (2009) 075.

[17] C. Keeler, G. Knodel, and J. T. Liu, J. High Energy Phys. 01 (2014) 062.

[18] C. Keeler, J. High Energy Phys. 01 (2014) 067.
[19] T. Andrade and S. F. Ross, Classical Quantum Gravity 30, 065009 (2013).

[20] L. S. Campos, Mathematica notebook with computations of this paper, https://github.com/lissadesouzacampos/ Klein_Gordon_field_on_Lifshitz_topological_blackholes.

[21] R. B. Mann, Ann. Isr. Phys. Soc. 13, 311 (1997).

[22] S. A. Hartnoll, A. Lucas, and S. Sachdev, arXiv: 1612.07324.

[23] P. Hořava and C. M. Melby-Thompson, Gen. Relativ. Gravit. 43, 1391 (2011).

[24] S. F. Ross, Classical Quantum Gravity 28, 215019 (2011).

[25] A. Terras, Harmonic Analysis on Symmetric Spaces and Applications I (Springer-Verlag New York Inc., 1985), p. 360.

[26] N. Limić, J. Niederle, and R. Rączka, J. Math. Phys. (N.Y.) 8, 1079 (1967).

[27] A. Zettl, Sturm-Liouville Theory, (American Mathematical Society, Providence, 2005), p. 328.

[28] C. Dappiaggi, H. R. C. Ferreira, and B. A. Jurez-Aubry, Phys. Rev. D 97, 085022 (2018).

[29] NIST Digital Library of Mathematical Functions, https:// dlmf.nist.gov/15, Accessed: 2021-03-28.

[30] M. H. Christensen, J. Hartong, N. A. Obers, and B. Rollier, Phys. Rev. D 89, 061901 (2014).

[31] I. Stakgold and M. J. Holst, Green's Functions and Boundary Value Problems (John Wiley \& Sons, New York, 2011), p. 99.

[32] H. Sahlmann and R. Verch, Commun. Math. Phys. 214, 705 (2000).

[33] I. Khavkine and V. Moretti, arXiv:1412.5945. 
[34] B. S. Kay and R. M. Wald, Phys. Rep. 207, 49 (1991).

[35] C. Dappiaggi and A. Marta, Math. Phys. Anal. Geom. 24, 28 (2021).

[36] V. S. Barroso and J. P. M. Pitelli, Gen. Relativ. Gravit. 52, 29 (2020).
[37] T. Morley, P. Taylor, and E. Winstanley, Phys. Rev. D 103, 045007 (2021).

[38] H. Braviner, R. Gregory, and S. F. Ross, Classical Quantum Gravity 28, 225028 (2011).

[39] J. T. Liu and Z. Zhao, arXiv:1206.1047. 